\title{
Statistical Theory of Spin Relaxation and Diffusion in Solids
}

\author{
A. L. Kuzemsky \\ Bogoliubov Laboratory of Theoretical Physics, Joint Institute for Nuclear Research, \\ 141980 Dubna, Moscow Region, Russia. \\ E-mail: kuzemsky@theor.jinr.ru
}

(Received February 21, 2006; revised May 19, 2006)

A comprehensive theoretical description is given for the spin relaxation and diffusion in solids. The formulation is made in a general statistical-mechanical way. The method of the nonequilibrium statistical operator (NSO) developed by Zubarev is employed to analyze a relaxation dynamics of a spin subsystem. Perturbation of this subsystem in solids may produce a nonequilibrium state which is then relaxed to an equilibrium state due to the interaction between the particles or with a thermal bath (lattice). The generalized kinetic equations were derived previously for a system weakly coupled to a thermal bath to elucidate the nature of transport and relaxation processes. In this paper, these results are used to describe the relaxation and diffusion of nuclear spins in solids. The aim is to formulate a successive and coherent microscopic description of the nuclear magnetic relaxation and diffusion in solids. The nuclear spin-lattice relaxation is considered and the Gorter relation is derived. As an example, a theory of spin diffusion of the nuclear magnetic moment in dilute alloys (like $\mathrm{Cu}-\mathrm{Mn}$ ) is developed. It is shown that due to the dipolar interaction between host nuclear spins and impurity spins, a nonuniform distribution in the host nuclear spin system will occur and consequently the macroscopic relaxation time will be strongly determined by the spin diffusion. The explicit expressions for the relaxation time in certain physically relevant cases are given.

KEY WORDS: Transport processes; nonequilibrium statistical operator; kinetic equation; spin temperature; nuclear spin relaxation and diffusion; dilute alloys.

\section{INTRODUCTION}

For many years there has been considerable interest, experimental and theoretical, in relaxation processes occurring in various spin systems, 
especially the nuclear spin systems in solids and liquids. ${ }^{1-38}$ In ordinary spin resonance experiments, spins are subject to an applied magnetic field $h_{0}$ and make a precessional motion around it. Local fields produced by interactions of the spins with their environments act as relatively weak perturbations to the unperturbed precessional motion. In quantum-mechanical language, the external field gives rise to the Zeeman levels for each spin and the interactions are perturbations to these quantum states. In a nuclear-magnetic resonance (NMR) experiment, the nuclear spin system absorbs energy from the externally applied radio-frequency field and transfers it to the thermal bath or reservoir provided by the lattice through the spin lattice interaction. The coupled nuclear spins in a solid with very slow spin-lattice relaxation time $T_{1}$ comprise a quasi-isolated system which for many purposes can be treated by thermodynamic methods. The spin-spin relaxation time is denoted by $T_{2}$. The other system, called the lattice, contains all other degrees of freedom, phonons, translational motion of conduction electrons, etc. It is at a temperature $T$ that it is considered stable. A macroscopic approach to the description of magnetic relaxation was proposed by Bloch. ${ }^{3}$ He proposed a phenomenological equation describing the motion of nuclear-spin system subjected to both a static and a time varying magnetic field

$$
\frac{d \vec{M}}{d t}=\gamma \vec{M} \times \vec{h}-\frac{M_{x}}{T_{2}} \vec{i}-\frac{M_{y}}{T_{2}} \vec{j}+\frac{M_{0}-M_{z}}{T_{1}} \vec{k},
$$

where the external field $\vec{h}$ is taken to be of the form $\vec{h}=h_{0} \vec{k}+2 h_{1}(t) \cos \omega t \vec{i}$. This equation successfully describes a wide variety of magnetic resonance experiments, although to obtain a valid description of low-frequency phenomena, it is necessary to modify the original equation so that relaxation takes place toward the instantaneous magnetic field. In an NMR experiment, the absorption of energy from the applied rf field produces either an increase in the energy of the spin system or a transfer of energy from the spin system to the lattice. The latter process requires a time interval of the order of spin-lattice relaxation time $T_{1}$. The characteristic time $T_{2}$ determines the relaxation of the transversal spin components due to the spin-spin interactions.

The relaxation processes in spin systems have been investigated by a number of authors $8,14,20-23,25-28,33,37$ to obtain qualitative and quantitative information about irreversible spin-spin and spin-lattice processes in spin systems. The method of many of these papers was to develop an equation of motion for the reduced density matrix ${ }^{12,13,16,17}$ describing the spin system, and was found to be most useful when the perturbation responsible for the relaxation of the spin system had a very short correlation time. In the equation-of-motion approach, the specification of the initial conditions involves the assumption of 
some explicit form for the density matrix describing the system (the system includes both the spin and its surroundings, which in the case studied below will be the conduction electrons in a metal). This problem is very attractive from the point of view of irreversible statistical mechanics since a general model of magnetic resonance consists of a driven system of interest in interaction with a heat bath. Stenholm and ter Haar ${ }^{18}$ have analyzed the basic assumptions which are necessary for statistical-mechanical derivation of the Bloch equation and the role of the thermal bath.

An important concept in the interpretation of spin-lattice relaxation phenomena was provided by the thermodynamic theory of Ref. 1 . They considered the magnetic crystal to be composed of two subsystems, which could be assigned two different temperatures. One subsystem contained the magnetic degrees of freedom. The other subsystem, called the lattice, contains all other degrees of freedom. Then, the idea of spin temperature was extended and several distinct temperatures for magnetic subsystems (Zeeman, dipole-dipole, etc.) were introduced. ${ }^{32}$ (Note, however, some special exclusions. ${ }^{39}$ ) In general, the state of the total system to be composed of a few subsystems may be described approximately by a density matrix of the form

$$
\rho \sim \exp \left[-\left(H_{1} / k T_{1}\right)-\left(H_{2} / k T_{2}\right)-\left(H_{3} / k T_{3}\right) \ldots\right]
$$

with a number of quasi-invariant energies $\operatorname{Tr}\left(H_{i}\right)$ and a number of distribution parameters $T_{i}^{-1}$. Nuclear relaxation in weak applied fields was first treated by Redfield ${ }^{9}$ and Hebel and Slichter, ${ }^{10}$ using the idea of spin temperature. Redfield theory is the semiclassical density operator theory of spin relaxation.

It was Bloembergen ${ }^{5}$ who first formulated that the magnetization of spins in a rigid lattice could be spatially transported by means of the mutual flipping of neighboring spins due to dipole-dipole interaction. This idea permitted one to explain the significant influence of a small concentration of paramagnetic impurities on spin-lattice relaxation in ionic crystals. $\mathrm{He}$ used a quantum-mechanical treatment ( first-order perturbation theory) and showed that the transport equation for magnetization was a diffusion equation. In this simple approximation he calculated the diffusion constant $D$. In other words, we can roughly represent the relaxation dynamics as

$$
\begin{aligned}
\frac{\partial\left\langle I^{z}(\vec{r})\right\rangle}{\partial t} & =-A(\vec{r})\left[\left\langle I^{z}(\vec{r})\right\rangle-\left\langle I^{z}(\vec{r})\right\rangle^{0}\right]+D(\vec{r}) \nabla^{2}\left\langle I^{z}(\vec{r})\right\rangle \\
\frac{\partial\left\langle I^{z}(\vec{r})\right\rangle}{\partial t} & =-\frac{\left\langle I^{z}(\vec{r})\right\rangle-\left\langle I^{z}(\vec{r})\right\rangle^{0}}{T_{1}} \\
\frac{1}{T_{1}} & \propto \frac{1}{T_{1}^{S L}}+\frac{1}{T_{1}^{D}},
\end{aligned}
$$

where $I^{z}$ is the $z$-component of the nuclear spin operator. 
Since then, many authors have formulated the general theory of the spin relaxation processes in solids from the standpoint of statistical mechanics or irreversible thermodynamics. An improvement in the general formulation of the theory was achieved by Kubo and Tomita ${ }^{6}$ in their treatment of magnetic resonance absorption via a linear theory of irreversible processes. In this theory the important quantities are frequency-dependent susceptibilities, which are expressed in terms of spin correlation functions. Buishvili ${ }^{40}$ developed a quantum-statistical theory of the dynamic polarization of nuclei by taking into account diffusion of nuclear spins as well as dipole interaction of electron spins. Buishvili and Zubarev ${ }^{23}$ developed a successive theory of spin diffusion in crystals. The nuclear diffusion in diamagnetic solids with paramagnetic impurities was analyzed by the method of the statistical operator for nonequilibrium systems. The Bloembergen equation, ${ }^{5}$ whose coefficients are explicitly expressed through certain correlation functions, was obtained. The theory of nuclear spin diffusion in the ferromagnets of certain type was considered in Ref. 41. The theory of the dynamic polarization of nuclei and nuclear relaxation for the case of strong saturation was analyzed in Ref. 42. The influence of a strong NMR saturation on spin diffusion was considered in Ref. 43. The time of spin-lattice relaxation was calculated for nuclei when spin diffusion was taken into account under conditions of a strong NMR saturation. The influence of exchange interactions between nuclear spins on the dynamic polarization of nuclei was considered in Ref. 44. Borckmans and Walgraef ${ }^{45}$ formulated a theory of Zeeman and dipolar energy diffusion in paramagnetic spin systems in the frame the general theory of irreversible processes developed by Prigogine and co-workers. Buishvili and Giorgadze ${ }^{46}$ investigated general theory of spin diffusion within the nonequilibrium statistical operator (NSO) approach. A consistent quantum statistical investigation of saturation of a nonuniformly broadened EPR line was carried out in Ref. 47 by taking into account spectral diffusion and the dipole-dipole reservoir. Role of the flip-flip and flip-flop transitions for the dynamic polarization of nuclei was analyzed in Ref. 48. The theory of spinlattice relaxation in crystals with paramagnetic impurities was discussed in paper of Ref. 49. The analysis of the role of the interaction between a few subsystems for the construction of the nonequilibrium density matrix was discussed from a general point of view by Buishvili and Zviadadze. ${ }^{50}$ The application of the NSO method to the case of relaxation in dilute alloys has been considered by Fazleev. ${ }^{51,52}$ The influence of relation between thermal capacities of nuclear spin subsystem and the reservoir of electron spin-spin interaction on spin kinetics, especially in the low-temperature case when the spin polarization of subsystems are high enough was analyzed in detail by Tayurskii ${ }^{53}$ for the case of insulators. 
Robertson ${ }^{27}$ derived an equation of motion for the total magnetic moment of a system containing a single species of nuclear spins in an arbitrarily time-dependent external magnetic field. He derived a generalization of Bloch's phenomenological equation for a magnetic resonance. In papers, ${ }^{21,22}$ the general quantum-statistical-mechanical approach to the problem of spin resonance and relaxation, which utilized a projection operator technique was developed. From the Liouville equation for the combined system of the spin subsystem and the thermal bath a nonMarkoffian equation for the time development of the statistical density operator for the spin system alone was derived. The memory effects were taken into account in the application of the method of the statistical operator for nonequilibrium systems to magnetic relaxation problem by Nigmatullin and Tayurskii. ${ }^{54}$ Romero-Ronchin et al. ${ }^{55}$ used a projection operator technique for derivation of the Redfield equations. ${ }^{8}$ In their paper, ${ }^{55}$ the relaxation properties of a spin system weakly coupled to lattice degrees of freedom were described using an equation of motion for the spin density matrix. This equation was derived using a general weak coupling theory, which was previously developed. To second-order in the weak coupling parameter, the results are in agreement with those obtained by Bloch, Wangsness and Redfield, but the derivation does not make use of second-order perturbation theory for short times. The authors claim that the derivation can be extended beyond second-order and ensures that the spin density matrix relaxes to its exact equilibrium form to the appropriate order in the weak coupling parameter.

In this work, we present a complementary theory which examines the relaxation dynamics of a spin system in the approach of the NSO. It uses a general formalizm from a previous study for a system that is in contact with a thermal bath (a "lattice") and relax to the equilibrium state. The aim of this paper is to show how the general theory of irreversible processes allows a theoretical study of such phenomena without postulated equations of phenomenological assumptions. One of our purposes in this paper is to present a unified statistical mechanical treatment of spin relaxation and spin diffusion phenomena. The transport of nuclear spin energy in a lattice of paramagnetic spins with magnetic dipolar interaction plays an important role in many relaxation processes. In this paper the microscopic derivation of an expression for the longitudinal relaxation time of bulk metal nuclear spins by dilute local moments is performed taking into account spin diffusion processes within the NSO statistical operator approach.

In the next section, we establish the notation and briefly present the main ideas of the NSO approach. This section includes a short summary of the derivation of the generalized kinetic and rate equations with the NSO method. 
Sec. 2 serves as an extended introduction to the present paper. In Sec. 3, the dynamics of the nuclear spin system is analyzed. We consider the application of the established equations to the derivation of the relaxation equations for spin systems. Special attention is given to the problem of spin relaxation and diffusion in Sec. 4. The case of nuclear spin diffusion in dilute magnetic alloys is discussed in some detail in Sec. 4.2. The final Section contains some concluding remarks concerning the results obtained.

\section{BASIC NOTIONS}

The statistical mechanics of irreversible processes in solids, liquids, and complex materials like a soft matter are at the present time of much interest. ${ }^{56-59}$ The central problem of nonequilibrium statistical mechanics is to derive a set of equations, which describe irreversible processes from the reversible equations of motion. ${ }^{57,59}$ The consistent calculation of transport coefficients is of particular interest because one can get information on the microscopic structure of the condensed matter. During the last decades, a number of schemes have been concerned with a more general and consistent approach to transport theory. ${ }^{57,60-62}$ These approaches, each in its own way, lead us to substantial advances in the understanding of the nonequilibrium behavior of many-particle classical and quantum systems. This field is very active and there are many aspects to the problem. ${ }^{63}$ Our purpose here is to discuss the derivation, within the formalism of the NSO, ${ }^{60}$ of the generalized transport and kinetic equations. On this basis we have derived, by statistical mechanics methods, the kinetic equations for a system weakly coupled to a thermal bath. ${ }^{64}$ Our motivation for presenting this alternative derivation is based on the conviction that the NSO method provides some advantages in displaying the physics of the relaxation processes.

\subsection{Outline of the Nonequilibrium Statistical Operator Method}

In this section, we briefly recapitulate the main ideas of the NSO approach $^{60,64}$ for the sake of a self-contained formulation. The precise definition of the nonequilibrium state is quite difficult and complicated, and is not uniquely specified. Since it is virtually impossible and impractical to try to describe in detail the state of a complex macroscopic system in the nonequilibrium state, the method of reducing the number of relevant variables was widely used. A large and important class of transport processes can reasonably be modeled in terms of a reduced number of macroscopic relevant variables. There are different time scales and different sets of the relevant variables, ${ }^{65,66}$ e.g. hydrodynamic, kinetic, 
etc. The most satisfactory and workable approach to the construction of Gibbs-type ensembles for the nonequilibrium systems, as it appears to the writer, is the method of NSO developed by Zubarev. ${ }^{60}$ The NSO method permits one to generalize the Gibbs ensemble method to the nonequilibrium case and construct a NSO, which enables one to obtain the transport equations and calculate the kinetic coefficients in terms of correlation functions, and which, in the case of equilibrium, goes over to the Gibbs distribution. Although this method is well known, we shall briefly recall it, mostly in order to introduce the notation needed in the following.

The NSO method sets out as follows. The irreversible processes, which can be considered as a reaction of a system on mechanical perturbations can be analyzed by means of the method of linear reaction on the external perturbation. ${ }^{59}$ However, there is also a class of irreversible processes induced by thermal perturbations due to the internal inhomogeneity of a system. Among them we have, e.g., diffusion, thermal conductivity, and viscosity. In certain approximate schemes it is possible to express such processes by mechanical perturbations which artificially induce similar nonequilibrium processes. However, the fact is that the division of perturbations into mechanical and thermal ones is reasonable in the linear approximation only. In the higher approximations in the perturbation, mechanical perturbations can effectively lead to the appearance of thermal perturbations.

The NSO method permits one to formulate a workable scheme for description of the statistical mechanics of irreversible processes, which include the thermal perturbation in a unified and coherent fashion. To perform this, it is necessary to construct statistical ensembles representing the macroscopic conditions determining the system. Such a formulation is quite reasonable if we consider our system for a suitable large time. For these large times the particular properties of the initial state of the system are irrelevant and the relevant number of variables necessary for description of the system reduces substantially. 65

The basic hypothesis is that after small time-interval $\tau$ the nonequilibrium distribution is established. Moreover, it is supposed that it is weakly time-dependent by means of its parameter only. Then the statistical operator $\rho$ for $t \geqslant \tau$ can be considered as an "integral of motion" of the quantum Liouville equation

$$
\frac{\partial \rho}{\partial t}+\frac{1}{i \hbar}[\rho, H]=0 .
$$

Here $\partial \rho / \partial t$ denotes time differentiation with respect to the time variable on which the relevant parameters $F_{m}$ depend. It is important to note once again that $\rho$ depends on $t$ by means of $F_{m}(t)$ only. We may consider that 
the system is in thermal, material, and mechanical contact with a combination of thermal baths and reservoirs maintaining the given distribution of parameters $F_{m}$. For example, it can be the densities of energy, momentum, and particle number for the system which is macroscopically defined by given fields of temperature, chemical potential, and velocity. It is assumed that the chosen set of parameters is sufficient to characterize macroscopically the state of the system. The set of the relevant parameters are dictated by the external conditions for the system under consideration and, therefore, the term $\partial \rho / \partial t$ appears as the result of the external influence upon the system. Due to this influence precisely, the behavior of the system is nonstationary. In order to describe the nonequilibrium process, it is necessary also to choose the reduced set of relevant operators $P_{m}$, where $m$ is the index (continuous or discrete). In the quantum case, all operators are considered to be in the Heisenberg representation

$$
P_{m}(t)=\exp \left(\frac{i H t}{\hbar}\right) P_{m} \exp \left(\frac{-i H t}{\hbar}\right),
$$

where $H$ does not depend on the time. The relevant operators may be scalars or vectors. The equations of motions for $P_{m}$ will lead to the suitable "evolution equations". ${ }^{60}$ In the quantum case

$$
\frac{\partial P_{m}(t)}{\partial t}-\frac{1}{i \hbar}\left[P_{m}(t), H\right]=0
$$

The time argument of the operator $P_{m}(t)$ denotes the Heisenberg representation with the Hamiltonian $H$ independent of time. Then, we suppose that the state of the ensemble is described by a NSO, which is a functional of $P_{m}(t)$

$$
\rho(t)=\rho\left\{\ldots P_{m}(t) \ldots\right\} .
$$

Then $\rho(t)$ satisfies the Liouville equation (2). Hence the quasi-equilibrium ("local-equilibrium") Gibbs-type distribution will have the form

$$
\rho_{q}=Q_{q}^{-1} \exp \left(-\sum_{m} F_{m}(t) P_{m}\right),
$$

where the parameters $F_{m}(t)$ have the meaning of time-dependent thermodynamic parameters, e.g., of temperature, chemical potential, and velocity (for the hydrodynamic stage), or the occupation numbers of one-particle states (for the kinetic stage). The statistical functional $Q_{q}$ is defined by demanding that the operator $\rho_{q}$ be normalized and equal to

$$
Q_{q}=\operatorname{Tr} \exp \left(-\sum_{m} F_{m}(t) P_{m}\right) .
$$


This description is still very simplified. There are various effects, which can make the picture more complicated. The quasi-equilibrium distribution is not necessarily close to the stationary stable state. There exists another, completely independent method for choosing a suitable quasi-equilibrium distribution. ${ }^{56,57,61,67}$ For the state with the extremal value of the informational entropy ${ }^{57,61}$

$$
S=-\operatorname{Tr}(\rho \ln \rho),
$$

provided that

$$
\operatorname{Tr}\left(\rho P_{m}\right)=\left\langle P_{m}\right\rangle_{q}, \quad \operatorname{Tr} \rho=1
$$

it is possible to construct a suitable quasi-equilibrium ensemble. Then the corresponding quasi-equilibrium (or local equilibrium ) distribution has the form

$$
\begin{gathered}
\rho_{q}=\exp \left(\Omega-\sum_{m} F_{m}(t) P_{m}\right) \equiv \exp (-S(t, 0)), \\
\Omega=\ln \operatorname{Tr} \exp \left(-\sum_{m} F_{m}(t) P_{m}\right),
\end{gathered}
$$

where $S(t, 0)$ can be called the entropy operator. The form of the quasiequilibrium statistical operator was constructed so as to ensure that the thermodynamic equalities for the relevant parameters $F_{m}(t)$

$$
\frac{\delta \ln Q_{q}}{\delta F_{m}(t)}=\frac{\delta \Omega}{\delta F_{m}(t)}=-\left\langle P_{m}\right\rangle_{q}, \quad \frac{\delta S}{\delta\left\langle P_{m}\right\rangle_{q}}=F_{m}(t)
$$

are satisfied. It is clear that the variables $F_{m}(t)$ and $\left\langle P_{m}\right\rangle_{q}$ are thermodynamically conjugate. Here the notation used is $\langle\ldots\rangle_{q}=\operatorname{Tr}\left(\rho_{q} \ldots\right)$.

By definition a special set of operators should be constructed which depends on the time through the parameters $F_{m}(t)$ by taking the invariant part of the operators $F_{m}(t) P_{m}$ occurring in the logarithm of the quasi-equilibrium distribution, i.e.,

$$
\begin{aligned}
B_{m}(t)= & \overline{F_{m}(t) P_{m}}=\varepsilon \int_{-\infty}^{0} e^{\varepsilon t_{1}} F_{m}\left(t+t_{1}\right) P_{m}\left(t_{1}\right) d t_{1} \\
= & F_{m}(t) P_{m}-\int_{-\infty}^{0} d t_{1} e^{\varepsilon t_{1}}\left(F_{m}\left(t+t_{1}\right) \dot{P}_{m}\left(t_{1}\right)\right. \\
& \left.+\dot{F}_{m}\left(t+t_{1}\right) P_{m}\left(t_{1}\right)\right),
\end{aligned}
$$

where $(\varepsilon \rightarrow 0)$ and

$$
\dot{P}_{m}=\frac{1}{i \hbar}\left[P_{m}, H\right], \quad \dot{F}_{m}(t)=\frac{d F_{m}(t)}{d t} .
$$


The parameter $\varepsilon>0$ will be set equal to zero, but only after the thermodynamic limit has been taken. Thus, the invariant part is taken with respect to the motion with Hamiltonian $H$. The operation of taking the invariant part, of smoothing the oscillating terms, is used in the formal theory of scattering ${ }^{68}$ to set the boundary conditions, which exclude the advanced solutions of the Schrodinger equation. The $\overline{P_{m}(t)}$ will be called the integrals (or quasi-integrals) of motion, although they are conserved only in the limit $(\varepsilon \rightarrow 0)$. It is clear that for the Schrodinger equation such a procedure excludes the advanced solutions by choosing the initial conditions. In the present context this procedure leads to the selection of the retarded solutions of the Liouville equation.

It should be noted that the same calculations can also be made with a deeper concept, the methods of quasi-averages. ${ }^{60,69}$

$$
\frac{\partial \ln \rho_{\varepsilon}}{\partial t}+\frac{1}{i \hbar}\left[\ln \rho_{\varepsilon}, H\right]=-\varepsilon\left(\ln \rho_{\varepsilon}-\ln \rho_{q}\right),
$$

where $(\varepsilon \rightarrow 0)$ after the thermodynamic limit. The required NSO is defined as

$$
\rho_{\varepsilon}=\rho_{\varepsilon}(t, 0)=\overline{\rho_{q}(t, 0)}=\varepsilon \int_{-\infty}^{0} d t_{1} e^{\varepsilon t_{1}} \rho_{q}\left(t+t_{1}, t_{1}\right) .
$$

Hence the NSO can then be written in the form

$$
\begin{aligned}
\rho= & Q^{-1} \exp \left(-\sum_{m} B_{m}\right) \\
= & Q^{-1} \exp \left\{-\sum_{m} F_{m}(t) P_{m}+\sum_{m} \int_{-\infty}^{0} d t_{1} e^{\varepsilon t_{1}}\left[\dot{F}_{m}\left(t+t_{1}\right) P_{m}\left(t_{1}\right)\right.\right. \\
& \left.\left.+F_{m}\left(t+t_{1}\right) \dot{P}_{m}\left(t_{1}\right)\right]\right\} .
\end{aligned}
$$

Now we can rewrite the NSO in the following useful form:

$$
\begin{aligned}
\rho(t, 0) & =\exp \left(-\varepsilon \int_{-\infty}^{0} d t_{1} e^{\varepsilon t_{1}} \ln \rho_{q}\left(t+t_{1}, t_{1}\right)\right) \\
& =\exp \overline{\left(\ln \rho_{q}(t, 0)\right)} \equiv \exp \overline{(-S(t, 0))} .
\end{aligned}
$$

The average value of any dynamic variable $A$ is given by

$$
\langle A\rangle=\lim _{\varepsilon \rightarrow 0^{+}} \operatorname{Tr}(\rho(t, 0) A)
$$


and is, in fact, the quasi-average. The normalization of the quasi-equilibrium distribution $\rho_{q}$ will persists after taking the invariant part if the following conditions are required

$$
\operatorname{Tr}\left(\rho(t, 0) P_{m}\right)=\left\langle P_{m}\right\rangle=\left\langle P_{m}\right\rangle_{q}, \quad \operatorname{Tr} \rho=1 .
$$

Before closing this section, we shall mention some modification of the "canonical" NSO method which was proposed in Ref. 67 and which one has to take into account in a more accurate treatment of transport processes.

\subsection{Transport and Kinetic Equations}

It is well known that the kinetic equations are of great interest in the theory of transport processes. Indeed, as it was shown in the preceding section, the main quantities involved are the following thermodynamically conjugate values:

$$
\left\langle P_{m}\right\rangle=-\frac{\delta \Omega}{\delta F_{m}(t)}, \quad F_{m}(t)=\frac{\delta S}{\delta\left\langle P_{m}\right\rangle} .
$$

The generalized transport equations, which describe the time evolution of variables $\left\langle P_{m}\right\rangle$ and $F_{m}$ follow from the equation of motion for the $P_{m}$, averaged with the NSO (16). It reads

$$
\left\langle\dot{P}_{m}\right\rangle=-\sum_{n} \frac{\delta^{2} \Omega}{\delta F_{m}(t) \delta F_{n}(t)} \dot{F}_{n}(t), \quad \dot{F}_{m}(t)=\sum_{n} \frac{\delta^{2} S}{\delta\left\langle P_{m}\right\rangle \delta\left\langle P_{n}\right\rangle}\left\langle\dot{P}_{n}\right\rangle .
$$

The entropy production has the form

$$
\dot{S}(t)=\langle\dot{S}(t, 0)\rangle=-\sum_{m}\left\langle\dot{P}_{m}\right\rangle F_{m}(t)=-\sum_{n, m} \frac{\delta^{2} \Omega}{\delta F_{m}(t) \delta F_{n}(t)} \dot{F}_{n}(t) F_{m}(t) .
$$

These equations are the mutually conjugate and with Eq. (19) form a complete system of equations for the calculation of values $\left\langle P_{m}\right\rangle$ and $F_{m}$.

\subsection{System in Thermal Bath: Generalized Kinetic Equations}

In paper, ${ }^{64}$ we derived the generalized kinetic equations for the system weakly coupled to a thermal bath. Examples of such systems can be an atomic (or molecular) system interacting with the electromagnetic field it generates as with a thermal bath, a system of nuclear or electronic spins interacting with the lattice, etc. The aim was to describe the relaxation processes in two weakly interacting subsystems, one of which is in the 
nonequilibrium state and the other is considered as a thermal bath. The concept of thermal bath or heat reservoir, i.e., a system that has effectively an infinite number of degrees of freedom, was not formulated precisely. A standard definition of the thermal bath is a heat reservoir defining a temperature of the system environment. From a mathematical point of view, ${ }^{66}$ a heat bath is something that gives a stochastic influence on the system under consideration. In this sense, the generalized master equation ${ }^{70}$ is a tool for extracting the dynamics of a subsystem of a larger system by the use of a special projection techniques ${ }^{71}$ or special expansion technique. ${ }^{72}$ The problem of a small system weakly interacting with a heat reservoir has various aspects. Basic to the derivation of a transport equation for a small system weakly interacting with a heat bath is a proper introduction of model assumptions. We are interested here in the problem of derivation of the kinetic equations for a certain set of average values (occupation numbers, spins, etc.), which characterize the nonequilibrium state of the system.

The Hamiltonian of the total system is taken in the following form:

$$
H=H_{1}+H_{2}+V \text {, }
$$

where

$$
H_{1}=\sum_{\alpha} E_{\alpha} a_{\alpha}^{\dagger} a_{\alpha}, \quad V=\sum_{\alpha, \beta} \Phi_{\alpha \beta} a_{\alpha}^{\dagger} a_{\beta}, \quad \Phi_{\alpha \beta}=\Phi_{\beta \alpha}^{\dagger} .
$$

Here $H_{1}$ is the Hamiltonian of the small subsystem, and $a_{\alpha}^{\dagger}$ and $a_{\alpha}$ are the creation and annihilation second quantized operators of quasiparticles in the small subsystem with energies $E_{\alpha}, V$ is the operator of the interaction between the small subsystem and the thermal bath, and $H_{2}$ the Hamiltonian of the thermal bath which we do not write explicitly. The quantities $\Phi_{\alpha \beta}$ are the operators acting on the thermal bath variables.

We assume that the state of this system is determined completely by the set of averages $\left\langle P_{\alpha \beta}\right\rangle=\left\langle a_{\alpha}^{\dagger} a_{\beta}\right\rangle$ and the state of the thermal bath by $\left\langle H_{2}\right\rangle$, where $\langle\ldots\rangle$ denotes the statistical average with the NSO, which will be defined below.

We take the quasi-equilibrium statistical operator $\rho_{q}$ in the form

$$
\begin{array}{r}
\rho_{q}(t)=\exp (-S(t, 0)), \quad S(t, 0)=\Omega(t)+\sum_{\alpha \beta} P_{\alpha \beta} F_{\alpha \beta}(t)+\beta H_{2} \\
\Omega=\ln \operatorname{Tr} \exp \left(-\sum_{\alpha \beta} P_{\alpha \beta} F_{\alpha \beta}(t)-\beta H_{2}\right) .
\end{array}
$$

Here $F_{\alpha \beta}(t)$ are the thermodynamic parameters conjugated with $P_{\alpha \beta}$, and $\beta$ is the reciprocal temperature of the thermal bath. All the operators are 
considered in the Heisenberg representation. The NSO has the form

$$
\begin{gathered}
\rho(t)=\exp (-\overline{S(t, 0)}), \\
\overline{S(t, 0)}=\varepsilon \int_{-\infty}^{0} d t_{1} e^{\varepsilon t_{1}}\left(\Omega\left(t+t_{1}\right)+\sum_{\alpha \beta} P_{\alpha \beta} F_{\alpha \beta}(t)+\beta H_{2}\right) .
\end{gathered}
$$

The parameters $F_{\alpha \beta}(t)$ are determined from the condition $\left\langle P_{\alpha \beta}\right\rangle=\left\langle P_{\alpha \beta}\right\rangle_{q}$.

In the derivation of the kinetic equations we use the perturbation theory in a "weakness of interaction" and assume that the equality $\left\langle\Phi_{\alpha \beta}\right\rangle_{q}=0$ holds, while other terms can be added to the renormalized energy of the subsystem. For further considerations it is convenient to rewrite $\rho_{q}$ as

$$
\rho_{q}=\rho_{1} \rho_{2}=Q_{q}^{-1} \exp \left(-L_{0}(t)\right),
$$

where

$$
\begin{aligned}
\rho_{1} & =Q_{1}^{-1} \exp \left(-\sum_{\alpha \beta} P_{\alpha \beta} F_{\alpha \beta}(t)\right), \\
Q_{1} & =\operatorname{Tr} \exp \left(-\sum_{\alpha \beta} P_{\alpha \beta} F_{\alpha \beta}(t)\right), \\
\rho_{2} & =Q_{2}^{-1} e^{-\beta H_{2}}, \quad Q_{2}=\operatorname{Tr} \exp \left(-\beta H_{2}\right), \\
Q_{q} & =Q_{1} Q_{2}, \quad L_{0}=\sum_{\alpha \beta} P_{\alpha \beta} F_{\alpha \beta}(t)+\beta H_{2} .
\end{aligned}
$$

We now turn to the derivation of the kinetic equations. The starting point is the kinetic equations in the following implicit form:

$$
\frac{d\left\langle P_{\alpha \beta}\right\rangle}{d t}=\frac{1}{i \hbar}\left\langle\left[P_{\alpha \beta}, H\right]\right\rangle=\frac{1}{i \hbar}\left(E_{\beta}-E_{\alpha}\right)\left\langle P_{\alpha \beta}\right\rangle+\frac{1}{i \hbar}\left\langle\left[P_{\alpha \beta}, V\right]\right\rangle .
$$

We restrict ourselves to the second-order in powers of $V$ in calculating the r.h.s. of (31). Finally, we obtain the kinetic equations for $\left\langle P_{\alpha \beta}\right\rangle$ in the form $^{64}$

$$
\frac{d\left\langle P_{\alpha \beta}\right\rangle}{d t}=\frac{1}{i \hbar}\left(E_{\beta}-E_{\alpha}\right)\left\langle P_{\alpha \beta}\right\rangle-\frac{1}{\hbar^{2}} \int_{-\infty}^{0} d t_{1} e^{\varepsilon t_{1}}\left\langle\left[\left[P_{\alpha \beta}, V\right], V\left(t_{1}\right)\right]\right\rangle_{q} .
$$

The last term of the right-hand side of Eq.(32) can be called the generalized "collision integral". Thus, we can see that the collision term for the system weakly coupled to the thermal bath has a convenient form of the double commutator as for the generalized kinetic equations ${ }^{73}$ for the system with small interaction. It should be emphasized that the assumption about the model form of the Hamiltonian (22) is nonessential for 
the above derivation. We can start again with the Hamiltonian (22) in which we shall not specify the explicit form of $H_{1}$ and $V$. We assume that the state of the nonequilibrium system is characterized completely by some set of average values $\left\langle P_{k}\right\rangle$ and the state of the thermal bath by $\left\langle H_{2}\right\rangle$. We confine ourselves to such systems for which $\left[H_{1}, P_{k}\right]=\sum_{l} c_{k l} P_{l}$. Then we assume that $\langle V\rangle_{q} \simeq 0$, where $\langle\ldots\rangle_{q}$ denotes the statistical average with the quasi-equilibrium statistical operator of the form

$$
\rho_{q}=Q_{q}^{-1} \exp \left(-\sum_{k} P_{k} F_{k}(t)-\beta H_{2}\right)
$$

and $F_{k}(t)$ are the parameters conjugated with $\left\langle P_{k}\right\rangle$. Following the method used above in the derivation of equation (32), we can obtain the generalized kinetic equations for $\left\langle P_{k}\right\rangle$ with an accuracy up to terms, which are quadratic in interaction

$$
\frac{d\left\langle P_{k}\right\rangle}{d t}=\frac{i}{\hbar} \sum_{l} c_{k l}\left\langle P_{l}\right\rangle-\frac{1}{\hbar^{2}} \int_{-\infty}^{0} d t_{1} e^{\varepsilon t_{1}}\left\langle\left[\left[P_{k}, V\right], V\left(t_{1}\right)\right]\right\rangle_{q} .
$$

Hence (32) is fulfilled for the general form of the Hamiltonian of a small system weakly coupled to a thermal bath.

\subsection{System in Thermal Bath: Rate and Master Equations}

In Sec. 2.3, we have described the kinetic equations for $\left\langle P_{\alpha \beta}\right\rangle$ in the general form. Let us write down Eq. (32) in an explicit form. We rewrite the kinetic equations for $\left\langle P_{\alpha \beta}\right\rangle$ as

$$
\frac{d\left\langle P_{\alpha \beta}\right\rangle}{d t}=\frac{1}{i \hbar}\left(E_{\beta}-E_{\alpha}\right)\left\langle P_{\alpha \beta}\right\rangle-\sum_{\nu}\left(K_{\beta \nu}\left\langle P_{\alpha \nu}\right\rangle+K_{\alpha \nu}^{\dagger}\left\langle P_{\nu \beta}\right\rangle\right)+\sum_{\mu \nu} K_{\alpha \beta, \mu \nu}\left\langle P_{\mu \nu}\right\rangle .
$$

The following notation were used

$$
\begin{aligned}
& \frac{1}{i \hbar} \sum_{\mu} \int_{-\infty}^{0} d t_{1} e^{\varepsilon t_{1}}\left\langle\Phi_{\beta \mu} \phi_{\mu v}\left(t_{1}\right)\right\rangle_{q} \\
& =\frac{1}{2 \pi} \sum_{\mu} \int_{-\infty}^{+\infty} d \omega \frac{J_{\mu v, \beta \mu}(\omega)}{\hbar \omega-E_{\gamma}-E_{\delta}+i \varepsilon}=K_{\beta v}, \\
& \frac{1}{i \hbar} \int_{-\infty}^{0} d t_{1} e^{\varepsilon t_{1}}\left(\left\langle\Phi_{\mu \alpha} \phi_{\beta \nu}\left(t_{1}\right)\right\rangle_{q}+\left\langle\phi_{\mu \alpha}\left(t_{1}\right) \Phi_{\beta \nu}\right\rangle_{q}\right)
\end{aligned}
$$




$$
\begin{aligned}
& =\frac{1}{2 \pi} \int_{-\infty}^{+\infty} d \omega J_{\beta \nu, \mu \alpha}(\omega)\left(\frac{1}{\hbar \omega-E_{\beta}+E_{\nu}+i \varepsilon}-\frac{1}{\hbar \omega-E_{\alpha}-E_{\mu}-i \varepsilon}\right) \\
& =K_{\alpha \beta, \mu \nu} .
\end{aligned}
$$

Let us now remind ${ }^{60}$ that the correlation functions $\langle A B(t)\rangle$ and $\langle A(t) B\rangle$ can be expressed via their spectral weight function (or spectral intensity) $J(\omega)$

$$
\begin{aligned}
& F_{A B}\left(t-t^{\prime}\right)=\left\langle A(t) B\left(t^{\prime}\right)\right\rangle=\frac{1}{2 \pi} \int_{-\infty}^{+\infty} d \omega, \exp \left[i \omega\left(t-t^{\prime}\right)\right] J_{A B}(\omega), \\
& F_{B A}\left(t^{\prime}-t\right)=\left\langle B\left(t^{\prime}\right) A(t)\right\rangle=\frac{1}{2 \pi} \int_{-\infty}^{+\infty} d \omega \exp \left[i \omega\left(t^{\prime}-t\right)\right] J_{B A}(\omega) .
\end{aligned}
$$

The correlation functions $\left\langle\Phi_{\beta \mu} \phi_{\mu \nu}\left(t_{1}\right)\right\rangle_{q}$ and $\left\langle\phi_{\nu \mu}\left(t_{1}\right) \Phi_{\mu \alpha}\right\rangle_{q}$ are connected with their spectral intensities in the following way:

$$
\begin{aligned}
\left\langle\Phi_{\mu \nu} \phi_{\gamma \delta}(t)\right\rangle_{q} & =\frac{1}{2 \pi} \int_{-\infty}^{+\infty} d \omega J_{\gamma \delta, \mu \nu}(\omega) \exp \left[-i\left(\omega-\frac{E_{\gamma}-E_{\delta}}{\hbar}\right) t\right] \\
\left\langle\phi_{\mu \nu}(t) \Phi_{\gamma \delta}\right\rangle_{q} & =\frac{1}{2 \pi} \int_{-\infty}^{+\infty} d \omega J_{\gamma \delta, \mu \nu}(\omega) \exp \left[i\left(\omega+\frac{E_{\mu}-E_{v}}{\hbar}\right) t\right] .
\end{aligned}
$$

The above result is similar in structure to the Redfield equation for the spin density matrix ${ }^{8}$ when the external time-dependent field is absent. Indeed, the Redfield equation of motion for the spin density matrix has the form ${ }^{8}$

$$
\frac{\partial \rho^{\alpha \alpha^{\prime}}}{\partial t}=-i \omega_{\alpha \alpha^{\prime}} \rho^{\alpha \alpha^{\prime}}+\sum_{\beta \beta^{\prime}} R_{\alpha \alpha^{\prime} \beta \beta^{\prime}} \rho^{\beta \beta^{\prime}} .
$$

Here $\rho^{\alpha \alpha^{\prime}}$ is the $\alpha, \alpha^{\prime}$ matrix element of the spin density matrix, $\omega_{\alpha \alpha^{\prime}}=$ $\left(E_{\alpha}-E_{\alpha^{\prime}}\right) \hbar$, where $E_{\alpha}$ is energy of the spin state $\alpha$ and $R_{\alpha \alpha^{\prime} \beta \beta^{\prime}} \rho^{\beta \beta^{\prime}}$ is the "relaxation matrix". A sophisticated analysis and derivation of the Redfield equation for the density of a spin system immersed in a thermal bath was given in Ref. 74. A brief discussion of the derivation of the Redfield-type equations in an external field is given in Appendix A.

Returning to Eq.(35), it is easy to see that if one confines himself to the diagonal averages $\left\langle P_{\alpha \alpha}\right\rangle$ only, this equation may be transformed to give 


$$
\begin{aligned}
\frac{d\left\langle P_{\alpha \alpha}\right\rangle}{d t} & =\sum_{\nu} K_{\alpha \alpha, \nu v}\left\langle P_{\nu v}\right\rangle-\left(K_{\alpha \alpha}+K_{\alpha \alpha}^{\dagger}\right)\left\langle P_{\alpha \alpha}\right\rangle, \\
K_{\alpha \alpha, \beta \beta} & =\frac{1}{\hbar^{2}} J_{\alpha \beta, \beta \alpha}\left(\frac{E_{\alpha}-E_{\beta}}{\hbar}\right)=W_{\beta \rightarrow \alpha}, \\
K_{\alpha \alpha}+K_{\alpha \alpha}^{\dagger} & =\frac{1}{\hbar^{2}} \sum_{\beta} J_{\beta \alpha, \alpha \beta}\left(\frac{E_{\beta}-E_{\alpha}}{\hbar}\right)=W_{\alpha \rightarrow \beta} .
\end{aligned}
$$

Here $W_{\beta \rightarrow \alpha}$ and $W_{\alpha \rightarrow \beta}$ are the transition probabilities expressed in the spectral intensity terms. Using the properties of the spectral intensities, ${ }^{60}$ it is possible to verify that the transition probabilities satisfy the relation of the detailed balance

$$
\frac{W_{\beta \rightarrow \alpha}}{W_{\alpha \rightarrow \beta}}=\frac{\exp \left(-\beta E_{\alpha}\right)}{\exp \left(-\beta E_{\beta}\right)}
$$

Finally, we have

$$
\frac{d\left\langle P_{\alpha \alpha}\right\rangle}{d t}=\sum_{\nu} W_{\nu \rightarrow \alpha}\left\langle P_{\nu v}\right\rangle-\sum_{\nu} W_{\alpha \rightarrow v}\left\langle P_{\alpha \alpha}\right\rangle .
$$

This equation has the usual form of the Pauli master equation.

It is well known that "the master equation is an ordinary differential equation describing the reduced evolution of the system obtained from the full Heisenberg evolution by taking the partial expectation with respect to the vacuum state of the reservoirs degrees of freedom". The rigorous mathematical derivation of the generalized master equation ${ }^{70-72}$ is rather a complicated mathematical problem.

\section{DYNAMICS OF NUCLEAR SPIN SYSTEM}

In NMR one has a system of nuclei with magnetic moment $\vec{\mu}$ and spins $\vec{I}$, which are placed in a magnetic field $h_{0}$. The magnetic moment $\vec{\mu}$ and momentum of nuclei $\vec{J}=\hbar \vec{I}$ are related as $\vec{\mu}=\gamma_{n} \vec{J}=\gamma_{n} \hbar \vec{I}=g_{n} \eta \vec{I}$, where $\gamma_{n}$ is the gyromagnetic nuclear factor, $g_{n}$ is the nuclear spectroscopic factor, and $\eta=e \hbar / 2 M c$ is the nuclear magneton. If the spins are otherwise independent, their interaction with the imposed field produces a set of degenerate energy levels, which for a system of $\mathrm{N}$ spins are $(2 I+1)^{N}$ in number with the energy spacing $\hbar \omega_{n}=\mu h_{0} / I$. It should be noted that the method of NMR is most powerful and useful in diamagnetic materials. Metals may be studied, although there are some technical specific problems.

In a NMR experiment, the nuclear spin system absorbs energy from the externally applied radio-frequency field and transfers it to the ther- 
mal bath or reservoir provided by the lattice through the spin-lattice interactions. The latter process requires a time interval of the order of the spin-lattice relaxation time $T_{1}$. The term "lattice" is used here to denote the equilibrium heat reservoir with temperature $T$ associated with all degrees of freedom of the system other than those associated with the nuclear spins.

A great advantage of magnetic resonance method is that the nuclear spin system is only very weakly coupled to the other degrees of freedom of the complex system in which it resides and its thermal capacity is extremely small. It is, therefore, possible to cause the nuclear spin system itself to depart severely from thermal equilibrium while leaving the rest of the material essentially in thermal equilibrium. As a consequence, the disturbance of the system other than the nuclear spins could be ignored.

If the nuclei are in thermodynamic equilibrium with the material at temperature $T$ in a field $h_{0}$, a nuclear paramagnetic moment $M_{0}$ is produced in the direction of $h_{0}$ given by the Curie formula $M_{0} / h_{0}=n \mu^{2} / 3 k T$, $n$ is the number of nuclei per unit volume.

We can evidently disturb the system from equilibrium by applying radiation from outside with quanta of size $\hbar \omega_{n}$ and with suitable polarization. If the equilibrium distribution is disturbed and the population changed, the magnetization in the $z$-direction, $M_{z}$, is different from $M_{0}$, say $M_{z}^{h}$. If then left alone, $M_{z}$ reverts to $M_{0}$ and usually does so exponentially with time, i.e.

$$
M_{z}(t)=M_{0}-\left(M_{0}-M_{z}^{h}\right) \exp \left\{-\frac{t}{T_{1}}\right\} .
$$

The last expression serves to define the spin-lattice relaxation time, $T_{1}$, and is so called because the process involves exchange of magnetic orientation energy with thermal energy of other degrees of freedom (known conventionally as a lattice). All the interactions with the nucleus may contribute to the relaxation process so we must add all contributions to $1 / T_{1}$

$$
\frac{1}{T_{1}} \propto \frac{1}{T_{1 \alpha}}+\frac{1}{T_{1 \beta}}+\frac{1}{T_{1 \gamma}}+\ldots,
$$

where various contributions to relaxation due to various interactions have been added. The relaxation rates may be dominated by one or more different physical interactions, so that the observable power spectrum may be the Fourier transform of functions involving dipole-dipole correlations, electric field gradient-nuclear quadrupole moment correlations, etc.

The dipole-dipole interaction Hamiltonian $H_{d d}$ between the magnetic moments of nuclei may contribute significantly to the nuclear magnetic relaxation process. ${ }^{75}$ Consider an explicit interaction between the moments 
$\vec{\mu}_{1}$ and $\vec{\mu}_{2}$, which are distant by $\vec{r}_{12}$, from each other. Then the interaction is written as

$$
\begin{aligned}
H_{d d}= & \frac{\vec{\mu}_{1} \vec{\mu}_{2}}{r_{12}^{3}}-\frac{3\left(\vec{\mu}_{1} \vec{r}_{12}\right)\left(\vec{\mu}_{2} \vec{r}_{12}\right)}{r_{12}^{5}} \\
= & -\sqrt{\frac{4 \pi}{5}} \frac{1}{r_{12}^{3}}\left[2 \mu_{1}^{z} \mu_{2}^{z} Y_{2,0}-\left(\mu_{1}^{+} \mu_{2}^{-}+\mu_{1}^{-} \mu_{2}^{+}\right) Y_{2,0}\right. \\
& +\sqrt{3}\left(\mu_{1}^{+} \mu_{2}^{z}+\mu_{1}^{z} \mu_{2}^{+}\right) Y_{2,-1}+\sqrt{3}\left(\mu_{1}^{-} \mu_{2}^{z}+\mu_{1}^{z} \mu_{2}^{-}\right) Y_{2,1} \\
& \left.+\sqrt{6} \mu_{1}^{+} \mu_{2}^{+} Y_{2,-2}+\sqrt{6} \mu_{1}^{-} \mu_{2}^{-} Y_{2,2}\right],
\end{aligned}
$$

where $\mu^{ \pm}=\left(\mu^{x} \pm \mu^{y}\right) / \sqrt{2}$ and $Y_{2, m}$ denote the normalized spherical harmonics of the second degree expressed in the form

$$
\begin{aligned}
Y_{2, \pm 2} & =\sqrt{\frac{15}{32 \pi}} \sin ^{2} \theta_{12} \exp ( \pm 2 i \phi), \quad Y_{2, \pm 1}=\sqrt{\frac{15}{8 \pi}} \sin \theta_{12} \cos \theta_{12} \exp ( \pm i \phi) ; \\
Y_{2,0} & =\sqrt{\frac{5}{16 \pi}}\left(3 \cos ^{2} \theta_{12}-1\right) .
\end{aligned}
$$

The dipole-dipole coupling provides the dissipation mechanisms in the spin system. It acts as time dependent perturbations on the Zeeman energy levels, which results in the relaxation of the nuclear magnetization.

Thus, such a spin system can be described as a superposition of a number of subsystems. They are the Zeeman subsystem for each spin species and the dipole-dipole subsystem. A weak applied rf field can be considered as an additional subsystem. The coupling inside each subsystem is strong, whereas the coupling between subsystems is weak. As a consequence, the subsystems reach internal thermal equilibrium independently of each other and one can ascribe a temperature, an energy, an entropy, etc., to each of them. Let us note that the usual prediction of statistical mechanics that the temperatures of interacting subsystems become equal in equilibrium is a direct consequence of the conjecture that the total energy is the only analytic constant of the motion.

\subsection{The Hierarchy of Time Scales}

A case of considerable practical interest in connection with the phenomenon of resonance and relaxation is that of the hierarchy of time scales. In the standard situations the interaction between nuclear spins is weak as well as the interaction with the lattice is weak. As a result, in the NMR case the thermal bath variables change on the fast time scale characterized by $t_{\mathrm{Lc}}$ while the spin variables change on the slow time scale characterized by $\tau_{\mathrm{sr}}$. First of all, consider the most important concept of 
spin temperature. ${ }^{32}$ Actually, spin systems are never completely isolated and the concept of spin temperature is meaningful only if the rate $\tau_{0}^{-1}$ of achievement of internal equilibrium is much faster than the spin-lattice relaxation rate $T_{1}^{-1}$. For time intermediate between $\tau_{0}$ and $\tau_{\mathrm{sr}}$, the spin temperature exists and can be different from the lattice temperature $T$. The necessary condition for the applicability of spin temperature concept is then inequality $\tau_{0} \sim T_{2} \ll T_{1}$.

Characteristic times are long in comparison with the time of achievement of internal equilibrium in the lattice $t_{\mathrm{Lc}}$ but short compared to spin relaxation times $t_{\mathrm{Lc}}<t<\tau_{\mathrm{sr}}$. In this case, the second-order perturbation theory is valid in the weak spin-lattice coupling parameter. Usually, it is assumed that the time $t_{\mathrm{Lc}}$ is very short and $\tau_{0} \geqslant t_{\mathrm{Lc}}$. The restriction of ordinary perturbation theory generally applied is that it is valid when within the time interval considered the density matrix cannot change substantially. Argyres and Kelley ${ }^{22}$ removed the restriction $t_{\mathrm{Lc}}<\tau_{\mathrm{sr}}$ and derived an equation of motion for the spin density that depends on the history of the system. ${ }^{74}$

One of the essential virtues of the NSO method is that it focuses attention, at the outset, on the existence of different time scales. Suppose that the Hamiltonian of the spin system can be divided as $H=H_{0}+V$, where $H_{0}$ is the dominant part, and $V$ is a weak perturbation. The separation of the Hamiltonian into $H_{0}$ and $V$ is not unique and depends on the physical properties of the system under consideration. The choice of the operator $H_{0}$ determines a short time scale $\tau_{0}$. This choice is such that for times $t \gg \tau_{0}$ the nonequilibrium state of the system can be described with a reasonable accuracy by the average values of some finite set of the operators $P_{m}$ (3).

After the short time $\tau_{0}$, it is supposed that the system can achieve the state of an incomplete or quasi-equilibrium state. The main assumption about the quasi-equilibrium state is that it is determined completely by the quasi-integrals of motion which are the internal parameters of the system. The characteristic relaxation time of these internal parameters is much longer than $\tau_{0}$. Clearly then, that even if these quasi-integrals at the initial moment had no definitive equilibrium values, after the time $\tau_{0}$, at the quasi-equilibrium state, those parameters, which altered quickly became the functions of the external parameters and of the quasi-integrals of motion. It is essential that this functional connection does not depend on the initial values of the parameters. In other words, the operators $P_{m}$ are chosen so that they should satisfy the condition : $\left[P_{k}, H_{0}\right]=$ $\sum_{l} c_{k l} P_{l}$. It is necessary to write down the transport equations (20) for this set of "relevant" operators only. The equations of motion for the average of other "irrelevant" operators ( other physical variables) will be in some 
sense consequences of these transport equations. As for the "irrelevant" operators, which do not belong to the reduced set of the "relevant" operators $P_{m}$, relation $\left[P_{k}, H_{0}\right]=\sum_{l} c_{k l} P_{l}$ leads to the infinite chain of operator equalities. For times $t \leqslant \tau_{0}$ the nonequilibrium averages of these operators oscillate fast, while for times $t>\tau_{0}$ they become functions of the average values of the operators.

\subsection{Nuclear Spin-Lattice Relaxation}

At the earlier stage, the theory of spin relaxation was developed by means of quantum mechanical perturbation methods. Here the spin relaxation is studied by making use of the method of NSO. We discuss in this section an arbitrary nuclear spin system on a lattice in interaction with external fields and another system, ${ }^{75}$ to be taken eventually to act as a heat bath. The bath is considered as a quantum-mechanical system that remained in thermodynamic equilibrium while its exchange of energy with the spin system is taken into account. We consider the processes occurring after switching off the external magnetic field in a nuclear spin subsystem of a crystal. Let us consider the behavior of a spin system with the Hamiltonian $H_{n}$ weakly coupled by a time-independent perturbation $V$ to a thermal bath (temperature reservoir) or a crystal lattice with the Hamiltonian $H_{L}$.

The total Hamiltonian has the form

$$
H=H_{n}+H_{L}+V
$$

where

$$
H_{n}=-a \sum_{i} I_{i}^{z}, \quad a=\gamma_{n} h_{0}
$$

Here $I_{i}^{z}$ is the operator of the $z$-component of the spin at the site $i, h_{0}$ the time-independent external field applied in the $z$-direction, and $\gamma_{n}$ the gyromagnetic coefficient.

Now we introduce $b_{i \lambda}^{\dagger}$ and $b_{i \lambda}$ the creation and annihilation operators of the spin in the site $i$ with the $z$-component of the spin equal to $\lambda$, where $-I \leqslant \lambda \leqslant I$. Then we have

$$
I_{i}^{z}=\sum_{\lambda} \lambda b_{i \lambda}^{\dagger} b_{i \lambda}=\sum_{\lambda} \lambda n_{i \lambda}
$$

and, consequently,

$$
H_{n}=\sum_{i \lambda} E_{\lambda} n_{i \lambda}, \quad E_{\lambda}=-a \lambda
$$


Following Sec. 2.3, we write the Hamiltonian of the interaction as

$$
V=\sum_{i} \sum_{\mu, \nu} \Phi_{i v, i \mu} b_{i v}^{\dagger} b_{i \mu}, \quad \Phi_{i v, i \mu}=\Phi_{i \mu, i v}^{\dagger}
$$

Here $\Phi_{i v, i \mu}$ are the operators acting only on the "lattice" variables. The term "lattice" is used here to denote the equilibrium heat reservoir with temperature $T$ associated with all degrees of freedom of the system other than those associated with the nuclear spins. Then, in agreement with Eq. (27), we construct the quasi-equilibrium statistical operator

$$
\rho_{q}=\rho_{L} \bigotimes \rho_{n},
$$

where

$$
\begin{gathered}
\rho_{L}=Q_{L}^{-1} e^{-\beta H_{L}}, \quad Q_{L}=\operatorname{Tr} \exp \left(-\beta H_{L}\right), \\
\rho_{n}=Q_{n}^{-N} \exp \left(-\beta_{n}(t) H_{n}\right), \quad Q_{n}=\frac{\sinh \frac{\beta_{n}(t)}{2} a(2 I+1)}{\sinh \frac{\beta_{n}(t)}{2} a} .
\end{gathered}
$$

Here $\beta_{n}$ is the reciprocal spin temperature and $N$ is the total number of spins in the system.

We now turn to writing down the kinetic equations for average values $\left\langle n_{i \lambda}\right\rangle=\left\langle b_{i \lambda}^{\dagger} b_{i \lambda}\right\rangle$. We use the kinetic equation in the form (45)

$$
\frac{d\left\langle n_{i \lambda}\right\rangle}{d t}=\sum_{\nu} W_{\nu \rightarrow \lambda}(i i)\left\langle n_{i v}\right\rangle-\sum_{\nu} W_{\lambda \rightarrow v}(i i)\left\langle n_{i \lambda}\right\rangle,
$$

where

$$
\begin{aligned}
& W_{\lambda \rightarrow v}(i i)=\frac{1}{\hbar^{2}} J_{\Phi_{i v, i \lambda} \Phi_{i \lambda, i v}}\left(\frac{E_{\nu}-E_{\lambda}}{\hbar}\right), \\
& W_{\nu \rightarrow \lambda}(i i)=\frac{1}{\hbar^{2}} J_{\Phi_{i \lambda, i v} \Phi_{i v, i \lambda}}\left(\frac{E_{\lambda}-E_{v}}{\hbar}\right) .
\end{aligned}
$$

It can be shown that

$$
\left\langle n_{i \lambda}\right\rangle=\left\langle n_{\lambda}\right\rangle=Q_{n}^{-1} \exp \left[-\beta_{n} E_{\lambda}\right] .
$$

Then we obtain

$$
\frac{d\left\langle n_{\lambda}\right\rangle}{d t}=\sum_{\nu} W_{\nu \rightarrow \lambda}\left\langle n_{\nu}\right\rangle-\sum_{\nu} W_{\lambda \rightarrow v}\left\langle n_{\lambda}\right\rangle,
$$

where

$$
W_{\lambda \rightarrow \nu}=\frac{1}{N} \sum_{i} W_{\lambda \rightarrow \nu}(i i), \quad W_{\nu \rightarrow \lambda}=\frac{1}{N} \sum_{i} W_{\nu \rightarrow \lambda}(i i) .
$$


It is easily seen that

$$
W_{v \rightarrow \lambda}=\exp \left[\beta\left(E_{v}-E_{\lambda}\right)\right] W_{\lambda \rightarrow v} .
$$

Hence, for $\beta_{n}$ we find the equation

$$
\frac{d \beta_{n}}{d t}=\frac{1}{2} \frac{\sum_{\nu \lambda}(\lambda-v) W_{\lambda \rightarrow \nu}\left(1-\exp \left[-\left(\beta-\beta_{n}\right)\left(E_{\lambda}-E_{\nu}\right)\right]\right) \exp \left[-\beta_{n} E_{\lambda}\right]}{\frac{Q_{n}}{a} \frac{\partial^{2} \ln Q_{n}}{\partial \beta_{n}^{2}}} .
$$

In the derivation of Eq. (59) we took into account that $\left\langle I^{z}\right\rangle=\sum_{v} v\left\langle n_{v}\right\rangle$ and

$$
\frac{d\left\langle I^{z}\right\rangle}{d t}=-\frac{1}{a} \frac{d \beta_{n}}{d t} \frac{\partial^{2} \ln Q_{n}}{\partial \beta_{n}^{2}}=-\frac{1}{a} \frac{d \beta_{n}}{d t}\left(\left\langle\left(I^{z}\right)^{2}\right\rangle-\left\langle I^{z}\right\rangle^{2}\right) .
$$

In the high-temperature approximation $\left(\hbar \omega_{n} \ll k T\right)$ we obtain

$$
\frac{d \beta_{n}}{d t}=\frac{\beta-\beta_{n}}{T_{1}},
$$

where $T_{1}$ is the longitudinal time of the spin-lattice relaxation

$$
\frac{1}{T_{1}}=\frac{1}{2} \frac{\sum_{\nu \lambda}(\lambda-v)^{2} W_{\lambda \rightarrow v}}{\sum_{v}(v)^{2}}
$$

The above expression is the well-known Gorter relation. ${ }^{4,14,33,35}$

\section{SPIN DIFFUSION OF NUCLEAR MAGNETIC MOMENT}

The concept of spin diffusion was invoked by Bloembergen ${ }^{5}$ to explain the magnetic relaxation of nuclei in diamagnetic solids, which is due to the interaction of the nuclear spins with spin of a paramagnetic impurity ion. This theoretical approach was further developed in many works. $9,23,26,33,76,77$ In the previous section, we have discussed a simple calculation of the longitudinal nuclear spin relaxation time within the NSO approach. Here we shall extend this treatment in order to obtain a more sophisticated description of the spin dynamics. Let us, therefore, work out a general formula, using these ideas.

Consider a subsystem of interacting nuclear spins $\vec{I}$ of a crystal which interact with the external magnetic field $h_{0}$ and with other subsystems of a crystal. Our aim is to derive the evolution equation for the reciprocal spin temperature of the Zeeman spin subsystem $\beta_{n}(\vec{r}, t)$ which is relaxed to the 
equilibrium after switching off the external rf field. The total Hamiltonian has the form

$$
H=H_{n}+H_{d d}+H_{L}+V,
$$

where the Zeeman operator $H_{n}$ is given by

$$
H_{n}=-a \sum_{i} I_{i}^{z}, \quad a=\gamma_{n} h_{0} .
$$

It is convenient to rewrite $H_{n}$ in the following form:

$$
\mathcal{H}_{n}(\vec{r})=\sum_{i} I_{i}^{z} \hbar\left(\omega_{n}+\Omega_{i}\right) \delta\left(\vec{r}-\vec{r}_{i}\right) .
$$

Here $\Omega_{i} \ll \omega_{n}$ is effective renormalization of the "bare" nuclear spin energy $\hbar \omega_{n}$ due to the surrounding medium and will be written explicitly below; $H_{d d}$ is the operator of dipole-dipole interaction (46)

$$
H_{d d}=\frac{g_{1} g_{2} \eta^{2}}{r^{3}} \sum_{i j}\left\{\vec{I}_{i} \vec{I}_{j}-3\left(\vec{I}_{i} \hat{r}\right)\left(\vec{I}_{j} \hat{r}\right)\right\},
$$

where $r$ is the distance between the two spins and $\hat{r}=\vec{r}[|\vec{r}|]^{-1}$ is the unit vector in the direction joining them. It was shown ${ }^{32,33}$ that the so-called secular part of this operator was essential, and in the rest of the paper we will use the notation $H_{d d}$ for the secular part of the operator of dipoledipole interaction. It has the form ${ }^{32,33}$

$$
\begin{aligned}
H_{d d} & =\sum_{i \neq j} A_{i j}\left(I_{i}^{z} I_{j}^{z}-\frac{1}{4}\left(I_{i}^{+} I_{j}^{-}+I_{i}^{-} I_{j}^{+}\right)\right) \\
& =\sum_{i \neq j} A_{i j}\left(I_{i}^{z} I_{j}^{z}-\frac{1}{2} I_{i}^{+} I_{j}^{-}\right) .
\end{aligned}
$$

Here

$$
A_{i j}=\frac{\gamma_{n}^{2} \hbar}{2 r_{i j}^{3}}\left(1-3 \cos ^{2} \theta_{i j}\right)
$$

and $\theta_{i j}$ is the angle between $\overrightarrow{h_{0}}$ and $\overrightarrow{r_{i j}}$;

$H_{L}$ is the Hamiltonian of the a thermal bath and $V$ the operator of interaction between the nuclear spins and the lattice. Since our aim is to derive the equation for the relaxation of the Zeeman energy, we take the operators $\mathcal{H}_{n}(\vec{r})$ and $H_{d d}$ as the relevant variables which describe the 
nonequilibrium state. According to the NSO formalism, we now write the entropy operator (24) in the form

$$
\begin{aligned}
S(t, 0) & =\Omega(t)+\beta H_{L}+\beta_{d} H_{d d}+\int \beta_{n}(\vec{r}, t) \mathcal{H}_{n}(\vec{r}) d^{3} r, \\
\rho_{q}(t) & =\exp (-S(t, 0)),
\end{aligned}
$$

where $\beta_{d}$ and $\beta$ are the reciprocal temperature of dipole-dipole subsystem and the thermal bath, respectively. Then, within the formalism of NSO, as described above in Sec. 2.3, it is possible to derive the corresponding transport equations for the nonequilibrium averages $\left\langle\mathcal{H}_{n}(\vec{r})\right\rangle$ and $\left\langle H_{d d}\right\rangle$. Here we confine ourselves to the equation for the $\left\langle\mathcal{H}_{n}(\vec{r})\right\rangle$ since the equations for $\beta_{n}(\vec{r}, t)$ and $\beta_{d}$ are decoupled when the external rf field is equal to zero.

We need the relations

$$
\frac{d \mathcal{H}_{n}(\vec{r})}{d t}=\frac{1}{i \hbar}\left[\mathcal{H}_{n}(\vec{r}), V\right]+\frac{1}{i \hbar}\left[\mathcal{H}_{n}(\vec{r}), H_{d d}\right]=K_{n}(\vec{r})-\operatorname{div} \vec{J}(\vec{r}) .
$$

Here $K_{n}(\vec{r})$ is the source term and $\vec{J}(\vec{r})$ is the effective nuclear spin energy current

$$
\vec{J}(\vec{r})=\frac{1}{2 i} \sum_{k \neq l} A_{k l} \vec{r}_{k l}\left(\omega_{n}+\Omega_{l}\right) \delta\left(\vec{r}-\vec{r}_{k}\right) I_{k}^{+} I_{l}^{-} .
$$

Since $\Omega_{i} \ll \omega_{n}$, the approximate form of the current is

$$
\vec{J}(\vec{r}) \approx \frac{\omega_{n}}{2 i} \sum_{k \neq l} A_{k l} \vec{r}_{k l} \delta\left(\vec{r}-\overrightarrow{r_{k}}\right) I_{k}^{+} I_{l}^{-}
$$

The law of conservation of energy in the differential form can be written as (c.f. Ref. 23)

$$
\frac{d\left\langle\mathcal{H}_{n}(\vec{r})\right\rangle}{d t}=-\operatorname{div}\langle\vec{J}(\vec{r})\rangle+\left\langle K_{n}(\vec{r})\right\rangle .
$$

Following the method of calculation of Buishvili and Zubarev, ${ }^{23}$ we get

$$
\frac{\partial\left\langle\mathcal{H}_{n}(\vec{r})\right\rangle}{\partial t}=-\sum_{\mu \nu=1,2,3} \frac{\partial}{\partial x_{\mu}} L^{\mu \nu}(\vec{r}) \frac{\partial}{\partial x_{\nu}} \beta_{n}(\vec{r}, t)+\left(\beta_{n}(\vec{r}, t)-\beta\right) L_{1}(\vec{r}) .
$$

According to Eq. (65), we have treated $\left\langle I^{z}(t)\right\rangle$ as a continuum function of spatial variables so that when evaluated at the lattice site $j$, it is equal to 
$\left\langle I_{j}^{z}(t)\right\rangle$. Carrying out a Taylor series expansion ${ }^{20}$ of $\left\langle I^{z}(t)\right\rangle$ about the $k$ th lattice site and then evaluating the results at position $j$ yield

$$
\begin{aligned}
\left\langle I_{j}^{z}(t)\right\rangle \approx & \left\langle I_{k}^{z}(t)\right\rangle+\left.\sum_{\alpha=1}^{3} \frac{\partial}{\partial x^{\alpha}}\left\langle I^{z}(t)\right\rangle\right|_{k} x_{k j^{\alpha}} \\
& +\left.\frac{1}{2} \sum_{\alpha, \beta=1}^{3} \frac{\partial^{2}}{\partial x^{\alpha} \partial x^{\beta}}\left\langle I^{z}(t)\right\rangle\right|_{k} x_{k j^{\alpha}} x_{k j}+\ldots,
\end{aligned}
$$

where $x_{k j^{\alpha}}$ is the $\alpha$ coordinate $(\alpha=1,2,3)$ in an arbitrary Cartesian coordinate system for $\vec{r}_{k j}$, and $\partial /\left.\partial x^{\alpha}\left\langle I^{z}(t)\right\rangle\right|_{k}$ is the partial derivative of $\left\langle I^{z}(t)\right\rangle$ with respect to $x^{\alpha}$, evaluated at the lattice site $k$.

The generalized kinetic coefficients $L^{\mu \nu}(\vec{r})$ and $L_{1}(\vec{r})$ have the form

$$
L^{\mu \nu}(\vec{r})=\int_{-\infty}^{0} d t_{1} \int_{0}^{1} d \lambda \int d^{3} q\left\langle J_{\mu}(\vec{r}) \exp (-\lambda S(t, 0)) J_{v}\left(\vec{q}, t_{1}\right) \exp (\lambda S(t, 0))\right\rangle_{q},
$$

$$
L^{1}(\vec{r})=\int_{-\infty}^{0} d t_{1} \int_{0}^{1} d \lambda \int d^{3} q\left\langle K_{n}(\vec{r}) \exp (-\lambda S(t, 0)) K_{n}\left(\vec{q}, t_{1}\right) \exp (\lambda S(t, 0))\right\rangle_{q}
$$

The condition $\left\langle\mathcal{H}_{n}(\vec{r})\right\rangle=\left\langle\mathcal{H}_{n}(\vec{r})\right\rangle_{q}$ determines the connection of $\beta_{n}(\vec{r}, t)$ and $\left\langle\mathcal{H}_{n}(\vec{r})\right\rangle$. Equation (72) is the diffusion type equation. ${ }^{[78-80]}$ This equation describes more fully the local changes of the Zeeman energy due to the relaxation and transport processes in the system with the Hamiltonian (63). In its general form Eq. (72) is very complicated ${ }^{[78-80]}$ and to get a solution, various approximate schemes should be used.

\subsection{Evaluation of Spin Diffusion Coefficient}

Let us consider the calculation of the diffusion coefficient. The most obvious approximation to express the average $\left\langle\mathcal{H}_{n}(\vec{r})\right\rangle$ in terms of $\beta_{n}(\vec{r}, t)$ is the high-temperature approximation $\beta F_{n}(t) \ll 1$ or $\hbar \omega_{n} \ll k T$. As a rule, this approximation is well fulfilled in the NMR experiment. Making use of high-temperature expansion in Eq. (72) and taking into account that in this approximation

$$
\exp (-S(t, 0)) \approx \frac{1}{\operatorname{Tr}_{I} 1}\left(1-\int d^{3} r \beta_{n}(\vec{r}, t) \mathcal{H}_{n}(\vec{r})\right) \rho_{L},
$$


we get

$$
\frac{\partial \beta_{n}(\vec{r})}{\partial t}=\sum_{\mu \nu} \frac{\partial}{\partial x_{\mu}} D^{\mu \nu}(\vec{r}) \frac{\partial}{\partial x_{\nu}} \beta_{n}(\vec{r}, t)-\left(\beta_{n}(\vec{r})-\beta\right) R(\vec{r})
$$

or in a different form

$$
\frac{\partial \beta_{n}(\vec{r})}{\partial t}=D(\vec{r}) \Delta \beta_{n}(\vec{r})-\left(\beta_{n}(\vec{r})-\beta\right) R(\vec{r}) .
$$

Here $D(\vec{r})$ is the diffusion coefficient

$$
D(\vec{r})=-\frac{1}{2 \hbar^{2} \omega_{n}^{2} N(r)} \int_{-\infty}^{0} e^{\varepsilon t_{1}} d t_{1} \int d^{3} r_{1} \frac{\operatorname{Tr}_{I}\left\langle J(\vec{r}) J\left(\vec{r}_{1}, t_{1}\right)\right\rangle_{L}}{\operatorname{Tr}_{I}\left(I^{z}\right)^{2}},
$$

$N(r)=\sum_{k} \delta\left(\vec{r}-\vec{r}_{k}\right)$ being the nuclear spin density. The quantity $R(\vec{r})>0$ has the following form:

$$
R(\vec{r})=-\frac{1}{\hbar^{2} \omega_{n}^{2} N(r)} \int_{-\infty}^{0} e^{\varepsilon t_{1}} d t_{1} \int d^{3} r_{1} \frac{\operatorname{Tr}_{I}\left\langle K_{n}(\vec{r}) K_{n}\left(\vec{r}_{1}, t_{1}\right)\right\rangle_{L}}{\operatorname{Tr}_{I}\left(I^{z}\right)^{2}}
$$

Here the symbol $\langle\ldots\rangle_{L}=\operatorname{Tr}\left(\ldots \rho_{L}\right)$ implies the average over the equilibrium ensemble for lattice degrees of freedom.

\subsection{Host Nuclear Spin Diffusion in Dilute Alloys}

Spin diffusion is the transport of Zeeman energy or magnetization via the dipole-dipole interactions and it proved important both theoretically 5,9,25,45,46 and experimentally 9,81 in diamagnetic solids. We consider here another class of substances, the dilute alloys. ${ }^{82-84}$ The spin dynamics and relaxation of bulk metal nuclei by relatively dilute local moments in dilute alloys (e.g. $\mathrm{Cu}-\mathrm{Mn}$ ) was studied quite extensively, both theoretically $^{85-93}$ and experimentally. ${ }^{94-110}$. The description of spin relaxation in dilute alloys has certain specific features as compared with the homogeneous systems. For brevity we confine ourselves to the consideration of the bulk metal nuclei relaxation in dilute alloy. Due to the dipole-dipole interaction between a nuclear spin and an impurity spin, the relaxation rate may become nonuniform. It is more rapid for the spins that are close to impurity and is much slower for the distant nuclear spins. As a result, a nonuniform distribution in the bulk nuclear spin subsystem will occur and to describe spin relaxation consistently, the nuclear spin diffusion should be taken into account.

The Hamiltonian for nuclear and electronic interacting spin subsystems is

$$
H=H_{n}+H_{e}+H_{M}+H_{n e}+H_{M e}+H_{\text {dip }}
$$


Here index $n$ denotes the host nuclear spins, $M$ denotes spin of the magnetic impurities, and $e$ denotes the electron subsystem. In this section, when we refer to the host nuclear spin subsystem $H_{n}$ we put

$$
H_{n}=\sum_{i} I_{i}^{z} \hbar \omega_{n}+\sum_{i \neq j} A_{i j}\left(I_{i}^{z} I_{j}^{z}-\frac{1}{2} I_{i}^{+} I_{j}^{-}\right) .
$$

The Hamiltonian of electron subsystem is

$$
H_{e}=\sum_{k \sigma} \varepsilon_{k \sigma} a_{k \sigma}^{\dagger} a_{k \sigma}
$$

and

$$
H_{M}=\sum_{m} \hbar \omega_{M} S_{m}^{z}
$$

is the Hamiltonian of the impurity spins in the external magnetic field. The Hamiltonian of the interaction ${ }^{110}$ of nuclear spins and the spin density $\vec{\sigma}\left(\vec{R}_{i}\right)$ of the conduction electrons is

$$
H_{n e}=J_{n e} \sum_{i} \vec{I}_{i} \vec{\sigma}\left(\vec{R}_{i}\right), \quad J_{n e}=-\frac{8 \pi}{\hbar^{2} \gamma_{n} \gamma_{e}},
$$

where

$$
\sigma_{k}^{+}=\sum_{q} a_{q \uparrow}^{\dagger} a_{k+q \downarrow}, \quad \sigma_{-k}^{-}=\left(\sigma_{k}^{+}\right)^{\dagger}=\sum_{q} a_{k+q \downarrow}^{\dagger} a_{q \uparrow} .
$$

Interaction of the impurity spins $\vec{S}_{m}$ and the spin density of the itinerant carriers is given by the spin-fermion ${ }^{111,112}(s p-d(f))$ model Hamiltonian

$$
H_{M e}=J_{s d} \sum_{m} \overrightarrow{S_{m}} \vec{\sigma}\left(\overrightarrow{R_{m}}\right)
$$

The last part of the total Hamiltonian (80)

$$
H_{d i p}=\hbar \sum_{i m} \sum_{\mu \nu=x, y, z} \Phi_{i m}^{\mu \nu} I_{i}^{\mu} S_{m}^{\nu}
$$

is the Hamiltonian of the dipole-dipole and pseudo-dipolar interaction of nuclear and impurity spins. This interaction was described in detail in Refs. 94,95,113. The pseudo-dipolar interaction does not originate in crystalline anisotropy but in the tensor character of the dipolar interaction. ${ }^{95}$ Their expression for the pseudo-dipolar interaction is

$$
H_{n n}^{P D}=\sum_{i j}\left[\vec{I}_{i} \vec{I}_{j}-3 r_{i j}^{-2}\left(\vec{I}_{i} \overrightarrow{r_{i j}}\right)\left(\vec{I}_{j} \overrightarrow{r_{i j}}\right)\right] B_{i j}
$$


The Van Vleck Hamiltonian for a system with two magnetic ingredients ${ }^{95,113}$ includes the term

$$
\begin{aligned}
H_{d i p}= & \sum_{i>j}\left(\frac{g_{n}^{2} \eta^{2}}{r_{i j}^{3}}+\tilde{B}_{i j}\right)\left\{\vec{I}_{i} \vec{I}_{j}-3 r_{i j}^{-2}\left(\vec{I}_{i} \vec{r}_{i j}\right)\left(\vec{I}_{j} \vec{r}_{i j}\right)\right\} \\
& +\sum_{i m}\left(\frac{g_{n} g_{e} \eta^{2}}{r_{i m}^{3}}+\tilde{B}_{i m}\right)\left\{\vec{I}_{i} \vec{S}_{m}-3 r_{i j}^{-2}\left(\vec{I}_{i} \overrightarrow{r_{i m}}\right)\left(\vec{S}_{m} \overrightarrow{r_{i m}}\right)\right\} \\
& +\sum_{m>n}\left(\frac{g_{e}^{2} \eta^{2}}{r_{m n}^{3}}+\tilde{B}_{m n}\right)\left\{\vec{S}_{m} \vec{S}_{n}-3 r_{m n}^{-2}\left(\vec{S}_{i} \overrightarrow{r_{m n}}\right)\left(\vec{S}_{j} \overrightarrow{r_{m n}}\right)\right\} .
\end{aligned}
$$

The $\tilde{B}$ 's represent the pseudo-dipolar interaction

$$
B_{i j}=\frac{3}{2}\left(\tilde{B}_{i j}+\frac{g_{n}^{2} \eta^{2}}{r_{i j}^{3}}\right)\left(1-3 \cos ^{2} \theta_{i j}\right) .
$$

The later consists of three components of which we use in Eq.(86) the following one as the most essential ${ }^{113,95}$

$$
H_{M n}^{\mathrm{PD}}=\sum_{i m} B_{i m} \vec{I}_{i}\left(\vec{S}_{m}-\hat{r}_{i m}\left(\hat{r}_{i m} \vec{S}_{m}\right)\right)
$$

It was shown in Ref. 95 that for the large distance between the nuclear spin and the electron spin $B_{i m}$ has the form

$$
B_{i m} \approx B \frac{\cos \left(k_{F} r_{i m}+\phi_{B}\right)}{\left(2 k_{F} r_{i m}\right)^{3}} \text {. }
$$

Thus, in structure, the coefficient $B_{i m}$ is similar to the production of the contact potential and the spatial part of the RKKY interaction. ${ }^{114}$ As a rule, the pseudo-dipolar interaction is less than the contact interaction. The estimations give $B \sim 1 / 3 J_{n e}$ for ${ }^{205} \mathrm{Tl}$. It will be even more valid for copper since its mass is much less than for $T l$.

Now the expression for the Hamiltonian $H_{\text {dip }}$ can be rewritten as

$$
\begin{aligned}
H_{\mathrm{dip}}= & \gamma_{n} \gamma_{M} \hbar \sum_{i m} \frac{1}{r_{i m}^{3}}\left\{I_{i}^{z} \delta S_{m}^{z}\left(1-3 \cos ^{2} \theta_{i m}\right)\right. \\
- & \left.\frac{3}{2} \sin \theta_{i m} \cos \theta_{i m}\left[\exp \left(-i \phi_{i m}\right) I_{i}^{+} \delta S_{m}^{z}+\exp \left(i \phi_{i m}\right) I_{i}^{-} \delta S_{m}^{z}\right]\right\} \\
& \left\{1+B \frac{\cos \left(2 k_{F} r_{i m}+\phi_{B}\right)}{8 k_{F}^{3}}\right\} .
\end{aligned}
$$


Here we have introduced the mean field $\left\langle S_{m}^{z}\right\rangle$ and the fluctuating part of the impurity spin, namely $\delta S_{m}^{z}=S_{m}^{z}-\left\langle S_{m}^{z}\right\rangle$. By substituting this definition of $S_{m}^{z}$ into (84) rewritten in terms of the variable $\delta S_{m}^{z}$ we obtain

$$
\begin{aligned}
H_{n e}= & -\frac{8 \pi}{\hbar^{2} \gamma_{n} \gamma_{e}} \sum_{i p}\left(\vec{I}_{i} \vec{\sigma}_{p}\right) \delta\left(\vec{R}_{i}-\vec{r}_{p}\right) \\
= & J_{n e} \sum_{i p}\left[\left(I_{i}^{+} \sigma_{p}^{-}+I_{i}^{-} \sigma_{p}^{+}\right) \delta\left(\vec{R}_{i}-\overrightarrow{r_{p}}\right)\right. \\
& \left.+\left(\sigma_{p}^{z} \delta\left(\vec{R}_{i}-\overrightarrow{r_{p}}\right)-\left\langle\sigma_{p}^{z} \delta\left(\vec{R}_{i}-\overrightarrow{r_{p}}\right)\right\rangle\right) I_{i}^{z}\right],
\end{aligned}
$$

where

$$
\sum_{p} \vec{\sigma}\left(\vec{r}_{p}\right) \delta\left(\vec{R}_{i}-\overrightarrow{r_{p}}\right)=\sum_{k k^{\prime}} \sum_{s s^{\prime}}\left\langle s|\vec{\sigma}| s^{\prime}\right\rangle \psi_{k^{\prime}}^{*}(0) \psi_{k}(0) a_{k s}^{\dagger} a_{k^{\prime} s^{\prime}} .
$$

Now it is possible to write down explicitly the shift of the Zeeman frequency $\omega_{n}$ in (65) due to the mean-field renormalization $\Omega_{i}$ as

$$
\begin{aligned}
\Omega_{i}= & \gamma_{n} \gamma_{M} \hbar \sum_{m} \frac{1}{r_{i m}^{3}}\left\{1+B \frac{\cos \left(2 k_{F} r_{i m}+\phi_{B}\right)}{8 k_{F}^{3}}\right\}\left\langle S^{z}\right\rangle \\
& J_{n e} \sum_{p}\left\langle\sigma_{p}^{z} \delta\left(\vec{R}_{i}-\vec{r}_{p}\right)\right\rangle=\sum_{m} \Phi_{i m}^{z z}\left\langle S_{m}^{z}\right\rangle-J_{n e} \sum_{p}\left\langle\sigma_{p}^{z} \delta\left(\vec{R}_{i}-\vec{r}_{p}\right)\right\rangle .
\end{aligned}
$$

This shift of the Zeeman frequency $\left(\Omega_{i} \ll \omega_{n}\right)$ is the most essential for the evaluation of the coefficient of spin diffusion. ${ }^{33,37,76}$

\subsection{Spin Diffusion Coefficient in Dilute Alloys}

Here, we evaluate concrete expressions for the spin diffusion coefficient (78) for the dilute alloys system which is described by the Hamiltonian (80). Consider again the approximate equation (76) where the diffusion coefficient can be written as

$$
D^{\mu \nu} \approx \frac{\omega_{d}}{\hbar^{2} \sqrt{\pi}} \sum_{l} A_{r l}^{2}\left(r^{\mu}-r_{l}^{\mu}\right)\left(r^{\nu}-r_{l}^{\nu}\right) \exp \left[-\left(\Omega_{r}-\Omega_{l}\right)^{2} / 4\left(\omega_{d}\right)^{2}\right] .
$$

In the derivation of the above expression, to permit explicit calculations, the Gaussian approximation for the nuclear spin correlation function was used (see Appendix B). From Eqs. (76) and (94) it follows that in the process of the longitudinal nuclear spin relaxation, which is a function of position, there is a possibility to transport the nuclear magnetization (i.e. excess of nuclear spin density) due to the dipole-dipole interaction. It is clearly seen that the nuclei themselves do not move in the spin diffusion 
process. There is diffusion of the excess of the projection of the nuclear spin only.

To proceed further, consider the case when the concentration of the impurity spins is very low. In this case, for one impurity spin there is a big number of host nuclear spins which interact with it. In other words, this case corresponds to the effective single-impurity situation. Thus, we can place one impurity spin to the origin of the coordinate frame $(0,0,0)$. The vector $\vec{r}$ in Eq. (94) is then counted from this position. For a simple cubic crystalline system with the inversion center the symmetric tensor $D^{\mu \nu}(\vec{r})$ is reduced to the scalar $D(\vec{r})$. The coefficient $D(\vec{r})$ decreases with decreasing the distance $r$ when $r$ is small. This is related with the fact that Zeeman nuclear frequencies of the nuclei, which are close to the impurity, have substantially different values due to the influence of the local magnetic fields induced by the impurity spin. This circumstance hinders the flip-flop $\left(\Omega=\omega_{M}-\omega_{n}\right)$ transitions of neighboring nuclei since this transition does not conserve the total Zeeman energy of nuclear spins. (Let us remind that if we suppose that the spins $S$ are completely polarized and the nuclear spins $I$ are completely unpolarized, then the dipolar interaction permits simultaneous reversals of $S$ and $I$ in the opposite directions, or flip-flops, and also reversals in the same direction which is usually called flip-flips with $\Omega=\omega_{M}+\omega_{n}$ ). In expression (94) this tendency is described by the exponential factor. This exponential factor leads to the appearance of the so-called "diffusion barrier" around each impurity. Inside this diffusion barrier the diffusion of nuclear spin is hindered strongly. ${ }^{33,76}$

It can be seen that for the large distance from the impurity the frequency difference in Eq. (94) behaves as $\left(\Omega_{r}-\Omega_{l}\right) \ll \omega_{d}$, where $\omega_{d} \approx$ $6 \gamma_{n}^{2} \hbar a^{-3}$ is the dipolar line-width and $D(r)$ does not depend on $r$. In the opposite case, of small distance scale (near impurity) the frequency difference is big and the coefficient $D(r)$ decreases quickly with the distance to the impurity. Thus, it is convenient to introduce the effective radius of the diffusion barrier $\delta$, namely, a distance from the impurity for which the following definition holds:

$$
D(r)= \begin{cases}D, & \text { if } r>\delta, \\ 0, & \text { if } r<\delta .\end{cases}
$$

The constant $D$ is equal to $D=\omega_{d} / 3 \hbar^{2} \sqrt{\pi} \sum A_{k l}^{2} r_{k l}^{2}$.

Let us estimate the "size" of the diffusion barrier. Consider two neighboring nuclei which take up a position along the radius from the impurity. The distance between them is equal to the lattice constant $a$. In this case, the frequency shift is equal to $\left(\Omega_{\delta}-\Omega_{\delta+a}\right) \approx \omega_{d}$ and $\delta \approx$ $a \sqrt[4]{\left[\gamma_{M} / \gamma_{n}\left\langle S^{z}\right\rangle\right]}$ ( see Appendix B). 
Consider again the approximate Eq.(77) taking into account the diffusion barrier approximation (95). It can be rewritten in the form

$$
\frac{\partial \beta_{n}(\vec{r}, t)}{\partial t}=D \Delta \beta_{n}(\vec{r}, t)-\left(\beta_{n}(\vec{r}, t)-\beta\right)\left(R_{0}+R_{1}(\vec{r})+R_{2}(\vec{r})\right),
$$

where

$$
\begin{gathered}
R_{0}=\frac{2 J_{n e}^{2}}{\hbar^{2} 2 \pi} \sum_{k k^{\prime}} \sum_{p p^{\prime}} \psi_{k}^{*} \psi_{k^{\prime}} \psi_{p}^{*} \psi_{p^{\prime}} \int_{-\infty}^{\infty} d \omega f\left(\omega-\omega_{n}\right) G_{k k^{\prime} p p^{\prime}}^{0}(\omega),(97) \\
G_{k k^{\prime} p p^{\prime}}^{0}(\omega)=\int_{-\infty}^{\infty} d t \exp (i t \omega)\left\langle a_{k \downarrow}^{\dagger} a_{k^{\prime} \uparrow} a_{p \uparrow}^{\dagger}(t) a_{p^{\prime} \downarrow}(t)\right\rangle \\
R_{1}(\vec{r})=-\frac{4 J_{n e}}{\hbar 2 \pi} \sum_{k k^{\prime}} \sum_{m} \int_{-\infty}^{\infty} d \omega f\left(\omega-\omega_{n}\right) \operatorname{Re}\left(\psi_{k}^{*} \psi_{k^{\prime}} G_{k k^{\prime} m}^{1}(\omega) \Phi_{r m}^{+z}\right),(99) \\
G_{k k^{\prime} m}^{1}(\omega)=\int_{-\infty}^{\infty} d t \exp (i t \omega)\left\langle a_{k \uparrow}^{\dagger} a_{k^{\prime} \downarrow} S_{m}^{z}(t)\right\rangle
\end{gathered}
$$

and

$$
\begin{aligned}
R_{2}(\vec{r}) & =\frac{9}{2\left(\gamma_{n} \gamma_{M} \hbar\right)^{2}} \frac{1}{2 \pi} \sum_{m} \int_{-\infty}^{\infty} d \omega f\left(\omega-\omega_{n}\right) G_{m m}^{i}(\omega) Y_{m}, \\
G_{m m}^{i}(\omega) & =\int_{-\infty}^{\infty} d t \exp (i t \omega)\left\langle\delta S_{m}^{z} \delta S_{m}^{z}(t)\right\rangle, \\
Y_{m} & =\left\{1+\frac{B \cos \left(2 k_{F}\left|\vec{r}-\vec{r}_{m}\right|+\phi_{B}\right)}{8 k_{F}^{3}}\right\}^{2} \frac{\sin ^{2} \theta_{r m} \cos ^{2} \theta_{r m}}{\left|\vec{r}-\vec{r}_{m}\right|^{6}} .
\end{aligned}
$$

Here the function $f\left(\omega-\omega_{n}\right)$ is the NMR line-shape. The line-shape of the NMR spectrum ${ }^{115}$ arises from the variation of the local field at a given nucleus because of the interaction with nearby neighbors. The inhomogeneity of the applied magnetic field may also increase the width of the line. The contribution of the factor $R_{0}^{-1}$ leads to the generalized Korringa relaxation rate $^{116}$

$$
\frac{1}{T_{1}} \propto \frac{\pi k T}{\hbar}\left[\frac{8 \pi}{3} \gamma_{n} \hbar \chi_{p} \frac{M}{\mu_{e}}\left\langle\left|\psi_{F}(0)\right|^{2}\right\rangle\right]^{2} .
$$

Korringa $^{116}$ calculated the spin-lattice relaxation time $T_{1}$ in metals and showed that $T_{1}$ should be inversely proportional to temperature and should be related to the Knight shift (see also Ref. 117). Korringa nuclear 
spin-lattice relaxation occurs in a metal through the nucleus-electron interaction of contact type ${ }^{116}$

$$
\frac{8 \pi}{3}\left(\left|\gamma_{e}\right| \hbar \vec{s}\right)\left(\gamma_{n} \hbar \vec{I}\right)\left|\psi_{A}(0)\right|^{2} .
$$

The quantity $R_{1}$ is determined by the correlation of the electron and impurity spins and is highly anisotropic.

The quantity $R_{2}$ is related to the scattering of nuclear spins on the fluctuations of impurity spins. The last contribution is the most essential factor in the present context. This is related to the fact that the main characteristic features of the problem under consideration clearly manifest itself in the isotropic case which is considered in the majority of works. In the isotropic case $R_{1}=0$ and the contribution of $R_{2}$ can be expressed as

$$
\begin{aligned}
R_{2}(r) & =\sum_{m} C\left\{1+\frac{B \cos \left(2 k_{F}\left|\vec{r}-\vec{r}_{m}\right|+\phi_{B}\right)}{8 k_{F}^{3}}\right\}^{2} \frac{1}{\left|\vec{r}-\vec{r}_{m}\right|^{6}}, \\
C & =\frac{3}{5\left(\gamma_{n} \gamma_{M} \hbar\right)^{2}} \frac{1}{2 \pi} \int_{-\infty}^{\infty} d \omega f\left(\omega-\omega_{n}\right) G_{\mathrm{mm}}^{i}(\omega) .
\end{aligned}
$$

Nevertheless, even after simplifications described above, a solution of the diffusion equation is still a complicated problem. The main difficulty is the presence of the highly oscillating factor $\cos \left(2 k_{F}\left|\vec{r}-\vec{r}_{m}\right|+\phi_{B}\right)$. The role of this oscillating factor can be taken into account entirely by numerical calculations. For a qualitative rough estimation we consider the simplified case when $B \approx 0$. Then we can proceed following the method of calculation of Ref. 76. According to these calculations ${ }^{76}$ we find

$$
\frac{1}{T_{1}}=\left(R_{0}\right)^{-1}+4 \pi \mathrm{DNF} \text {. }
$$

Here $N$ is the number of impurities and the quantity $F$ has the form

$$
F= \begin{cases}0.7 b, & \text { if } b>\delta, \\ 1 / 3(b / \delta)^{3} b, & \text { if } b<\delta,\end{cases}
$$

where $b=\sqrt[4]{(C / D)}$.

It is clear from Eqs. (108) and (109) that the behavior of the relaxation time and its value depend strongly on the interrelation of $b$ which is determined by the correlation function $G_{m m}^{i}(\omega)$ and of $\delta$ which is determined by $\left\langle S^{z}\right\rangle$, as well as on the temperature for each concrete alloy. Thus, the problem of description of spin-lattice relaxation in dilute metallic alloys was reduced to the problem of calculation of the value of $F$. When $\delta \ll b$ the diffusion barrier is nonessential. In the opposite case, when $b<\delta$, the 
diffusion barrier is essential and leads to the slowing down of the relaxation process. In other words, the distance $b$ determines the scale up to which the nuclear spin relaxation is effective. Finally, let us note that the order of value of time which is necessary to transmit the magnetic moment to the distance $r$ in a solid is equal to $\tau_{D} \simeq r^{2} / D$; for $r=10^{-6} \mathrm{~cm}$ it gives the value $\tau_{D} \simeq 1 \mathrm{sec}$.

\section{CONCLUDING REMARKS}

In the present paper, we have given a complementary method for obtaining the rate and relaxation equations of nuclear spin system in solids. The main tool in this approach is the use of the method of NSO. ${ }^{60]}$ We have presented a theory of spin relaxation which allows us to derive general equations of spin dynamics. In addition, our theory allows us to take into account the effects of spin diffusion in a very straightforward manner. The calculations were kept general by restricting the form of spinlattice Hamiltonian as little as possible. It has permitted us to perform the derivation under more general conditions and explicitly demonstrate some key features of irreversible processes in solids.

It was shown that the spin systems provide a useful proving ground for applying the sophisticated methods of statistical thermodynamics. The method used is capable of systematic improvement and gives a deeper insight into the meaning of the spin relaxation processes in solids. We have shown that the transport of nuclear spin energy in a lattice of paramagnetic spins with magnetic dipolar interaction plays an important role in relaxation processes in solids. To test the general formalism presented here, an example of a dilute metallic alloy system was considered to demonstrate the usefulness of the equations derived.

In summary, the present paper examines the relaxation dynamics of a spin system. It continues the investigation presented in the previous work into the use of statistical mechanical methods for systems that are in contact with a thermal bath. We used the method of the NSO developed by Zubarev. In the present paper, we have developed the application of this method to the spin-relaxation problem, so that some useful results may be obtained from it. The calculation presented in this paper can be said to show that the NSO method has provided a compact and efficient tool for description of the spin relaxation dynamics. In this respect, the present treatment may be regarded as a complement of the Buishvili and Zubarev $^{23}$ seminal treatment.

Though the analysis of this paper concentrates on the nuclear spin systems in solids, the extension to other spin systems, e.g., paramagnetic electron spin system, is straightforward. The other important task is to 
examine the effects of a periodically time-dependent field on the longtime behavior of an otherwise isolated system of many coupled spins. This question is a part of a more general problem of the evolution of a complex system in an external field, especially in an intense external field. We hope that the methods here developed may be applied in these cases with the suitable modifications.

\section{APPENDIX A. EVOLUTION OF A SYSTEM IN AN ALTERNATING EXTERNAL FIELD}

In Sec. the 2.2 we wrote the kinetic and evolution equations in the approach of NSO. In this appendix, we show briefly the derivation of the same equations in the presence of alternating external field. This problem is essential for the nuclear and electron spin resonance. Both nuclear and electron spins have associated magnetic dipole moments, which can absorb radiation, usually at radio or microwave frequencies.

We consider the many-particle system with the Hamiltonian

$$
H=H_{1}+H_{2}+V+H_{f}(t),
$$

where

$$
H_{1}=\sum_{\alpha} E_{\alpha} a_{\alpha}^{\dagger} a_{\alpha}
$$

is the single-particle second-quantized Hamiltonian of the quasiparticles with energies $E_{\alpha}$. This term corresponds to the kinetic energy of noninteracting particles

$$
H_{1}=\sum_{i=1}^{N} \frac{P_{i}^{2}}{2 m}=\sum_{i=1}^{N} H(i), \quad H(i)=-\frac{\hbar^{2}}{2 m} \nabla_{i}^{2} .
$$

The index $\alpha \equiv(\vec{k}, s)$ denotes the momentum and spin

$$
\begin{gathered}
\varphi_{\alpha}(x)=\varphi_{\vec{k}}(\vec{r}) \Delta(s-\sigma)=\exp (i \vec{k} \vec{r}) \Delta(s-\sigma) / \sqrt{v}, \\
E_{\alpha}=\left\langle\alpha\left|H_{1}\right| \alpha\right\rangle, \\
\left\langle k|H(1)| k^{\prime}\right\rangle=\frac{1}{v} \int d^{3} r \exp (i \vec{k} \vec{r})\left(-\frac{\hbar^{2}}{2 m} \nabla^{2}\right) \exp \left(i \overrightarrow{k^{\prime}} \vec{r}\right)=\frac{\hbar^{2} k^{2}}{2 m} \Delta\left(k-k^{\prime}\right)
\end{gathered}
$$

and

$$
V=\sum_{\alpha, \beta} \Phi_{\alpha \beta} a_{\alpha}^{\dagger} a_{\beta}, \quad \Phi_{\alpha \beta}=\Phi_{\beta \alpha}^{\dagger}
$$


Operator $V$ is the operator of the interaction between the small subsystem and the thermal bath, and $\mathrm{H}_{2}$ the Hamiltonian of the thermal bath which we do not write explicitly. The quantities $\Phi_{\alpha \beta}$ are the operators acting on the thermal bath variables with the properties $\left(\Phi_{\alpha \beta}\right)^{\dagger}=\Phi_{\beta \alpha}^{*} ; \Phi_{\beta \alpha}^{*}=\Phi_{\alpha \beta}$. The interaction of the system with the external time dependent alternating field is described by the operator

$$
H_{f}(t)=\sum_{\alpha, \beta} h_{\alpha \beta}(t) a_{\alpha}^{\dagger} a_{\beta} .
$$

For purposes of calculation, it is convenient to rewrite Hamiltonian $H_{f}(t)$ in a somewhat different form

$$
H_{f}(t)=\frac{1}{v} \sum_{\alpha, \beta} T(\alpha, \beta, t) a_{\alpha}^{\dagger} a_{\beta},
$$

where

$$
h_{\alpha \beta}(t)=\frac{1}{v} T(\alpha, \beta, t)
$$

and

$$
\begin{gathered}
T=\sum_{i=1}^{N} T\left(\overrightarrow{r_{i}}, t\right) ; \quad T(\vec{p})=\int d^{3} r \exp (i \vec{p} \vec{r}) T(\vec{r}, t), \\
\left\langle k|T(\vec{r}, t)| k^{\prime}\right\rangle=\frac{1}{v} \int d^{3} r \exp \left(i\left(\vec{k}-\vec{k}^{\prime}\right) \vec{r}\right) T(\vec{r}, t)=\frac{1}{v} T\left(\vec{k}-\vec{k}^{\prime}, t\right) .
\end{gathered}
$$

We are interested in the kinetic stage of the nonequilibrium process in the system weakly coupled to the thermal bath. Therefore, we assume that the state of this system is determined completely by the set of averages $\left\langle P_{\alpha \beta}\right\rangle=$ $\left\langle a_{\alpha}^{\dagger} a_{\beta}\right\rangle$ and the state of the thermal bath by $\left\langle H_{2}\right\rangle$, where $\langle\ldots\rangle$ denotes the statistical average with the NSO, which will be defined below.

Following Pokrowsky's calculations we can write down the NSO in the following form:

$$
\rho(t)=Q^{-1} \exp (-L(t)),
$$

where

$$
\begin{aligned}
L(t)= & \sum_{\alpha \beta} P_{\alpha \beta} F_{\alpha \beta}(t)+\beta \mathcal{H}_{2}-\int_{-\infty}^{0} d t_{1} e^{\varepsilon t_{1}} \\
& \times\left(\sum_{\alpha \beta} \dot{P}_{\alpha \beta}\left(t_{1}\right) F_{\alpha \beta}\left(t+t_{1}\right)+\sum_{\alpha \beta} P_{\alpha \beta}\left(t_{1}\right) \frac{\partial F_{\alpha \beta}\left(t+t_{1}\right)}{\partial t_{1}}+\beta J_{2}\right) .
\end{aligned}
$$


The notation $\mathcal{H}_{2}$ denotes $\mathcal{H}_{2}=H_{2}-\mu_{2} N_{2}$ where $\mu_{2}$ is the chemical potential of the medium (thermal bath) and $J_{2}=\dot{\mathcal{H}}_{2}\left(t_{1}\right)$. In this equation, the time dependence of the operators in the right-hand side differs from the time dependence in Eq. (25). Consider this question in detail. The Heisenberg representation

$$
H(t)=U^{\dagger}(t) H U(t) ; \quad U(t)=\exp \left(\frac{-i H t}{\hbar}\right)
$$

in the presence of the external field $H=H_{0}+H^{\text {ext }}$ takes the form

$$
A\left(t_{1} ; t\right)=U^{\dagger}\left(t+t_{1} ; t\right) A U\left(t+t_{1} ; t\right),
$$

where

$$
U\left(t+t_{1} ; t\right)=T \exp \left(-\frac{i}{\hbar} \int_{t}^{t+t_{1}} H^{\mathrm{ext}}(\tau) d \tau\right)
$$

We now generalize the evolution equations to the case in which the external field is present. We have

$$
\begin{aligned}
\dot{P}_{\alpha \beta}= & \frac{1}{i \hbar}\left[P_{\alpha \beta}, H\right]=\frac{1}{i \hbar}\left(E_{\beta}-E_{\alpha}\right) P_{\alpha \beta} \\
& +\frac{1}{i \hbar} \sum_{\nu}\left(P_{\alpha \nu} h_{\beta \nu}(t)-h_{\nu \alpha}(t) P_{\nu \beta}\right)+\frac{1}{i \hbar}\left[P_{\alpha \beta}, V\right] .
\end{aligned}
$$

Then we can write down the balance equation

$$
J_{1}+J_{2}=I_{f},
$$

where

$$
J_{1}=\dot{H}_{1}=\frac{1}{i \hbar}\left[H_{1}, H\right]=\frac{1}{i \hbar}\left(\left[H_{1}, V\right]+\left[H_{1}, H^{\mathrm{ext}}\right]\right)
$$

and

$$
I_{f}=J_{1}+J_{2}=\frac{1}{i \hbar} \sum_{\alpha \beta}\left\{\left(E_{\alpha}-E_{\beta}\right) h_{\alpha \beta}(t)+h_{\alpha \beta}(t)\left[P_{\alpha \beta}, V\right]\right\} .
$$

The last term describes the work of the external field.

The parameters $F_{\alpha \beta}(t)$ are determined from the condition $\left\langle P_{\alpha \beta}\right\rangle=\left\langle P_{\alpha \beta}\right\rangle_{q}$. The quasi-equilibrium statistical operator $\rho_{q}$ has the form

$$
\rho_{q}=\rho_{1} \rho_{2},
$$


where

$$
\begin{gathered}
\rho_{1}=Q_{1}^{-1} \exp \left(-\sum_{\alpha \beta} P_{\alpha \beta} F_{\alpha \beta}(t)\right), Q_{1}=\operatorname{Tr} \exp \left(-\sum_{\alpha \beta} P_{\alpha \beta} F_{\alpha \beta}(t)\right), \\
\rho_{2}=Q_{2}^{-1} \exp \left(-\beta\left(H_{2}-\mu_{2} N_{2}\right)\right), Q_{2}=\operatorname{Tr} \exp \left(-\beta\left(H_{2}-\mu_{2} N_{2}\right)\right) .
\end{gathered}
$$

Thus, we can write

$$
\begin{aligned}
\frac{d\left\langle P_{\alpha \beta}\right\rangle}{d t}= & \frac{1}{i \hbar}\left\langle\left[P_{\alpha \beta}, H\right]\right\rangle=\frac{1}{i \hbar}\left(E_{\beta}-E_{\alpha}\right)\left\langle P_{\alpha \beta}\right\rangle \\
& +\frac{1}{i \hbar} \sum_{v}\left(\left\langle P_{\alpha \nu}\right\rangle h_{\beta v}(t)-h_{\nu \alpha}(t)\left\langle P_{\nu \beta}\right\rangle\right)+\frac{1}{i \hbar}\left\langle\left[P_{\alpha \beta}, V\right]\right\rangle .
\end{aligned}
$$

To calculate explicitly the r.h.s. of Eq. (127) we use the formula

$$
\begin{aligned}
& \exp (-A-B)=\exp (-A)-\int_{0}^{1} \exp (-A)(\exp (-A \tau) B \exp (A \tau) d \tau) \\
& \left.\rho \simeq\left\{1-\int_{0}^{1}\left(\exp (-A \tau) B \exp (A \tau)-\langle\exp (-A \tau) B \exp (A \tau)\rangle_{A}\right) d \tau\right)\right\} \rho(A)
\end{aligned}
$$

where

$$
\begin{aligned}
\rho(A) & =\frac{\exp (-A)}{\operatorname{Tr} \exp (-A)}, \\
A & =\sum_{\alpha \beta} P_{\alpha \beta} F_{\alpha \beta}(t)+\beta\left(H_{2}-\mu_{2} N_{2}\right), \\
B & =-\int_{-\infty}^{0} d t_{1} e^{\varepsilon t_{1}} \frac{1}{i \hbar} \sum_{\mu \nu}\left[P_{\mu \nu}\left(t_{1}, t\right), V\left(t_{1}\right)\right] X_{\mu \nu}\left(t+t_{1}\right) .
\end{aligned}
$$

Making the same expansion procedure as described in Sec. 2.2 we find

$$
\begin{aligned}
\frac{d\left\langle P_{\alpha \beta}\right\rangle}{d t}= & \frac{1}{i \hbar}\left(E_{\beta}-E_{\alpha}\right)\left\langle P_{\alpha \beta}\right\rangle+\frac{1}{i \hbar} \sum_{\nu}\left(\left\langle P_{\alpha \nu}\right\rangle h_{\beta \nu}(t)-h_{\nu \alpha}(t)\left\langle P_{\nu \beta}\right\rangle\right) \\
& +\frac{\beta}{i \hbar} \int_{0}^{1} d \lambda\left\langle\left[P_{\alpha \beta}, V\right] e^{-\lambda A} V e^{\lambda A}\right\rangle_{q}+\frac{1}{(i \hbar)^{2}} \int_{0}^{1} d \lambda \int_{-\infty}^{0} d t_{1} e^{\varepsilon t_{1}} \\
& \times \sum_{\alpha^{\prime} \beta^{\prime}}\left\langle\left[P_{\alpha^{\prime} \beta^{\prime}}, V\right] e^{-\lambda A}\left[P_{\alpha^{\prime} \beta^{\prime}}\left(t_{1}, t\right), V\left(t_{1}\right)\right] e^{\lambda A}\right\rangle_{q} X_{\alpha^{\prime} \beta^{\prime}}\left(t+t_{1}\right),(1
\end{aligned}
$$

where

$$
X_{\alpha^{\prime} \beta^{\prime}}(t)=F_{\alpha^{\prime} \beta^{\prime}}(t)-\beta\left[\delta_{\alpha^{\prime} \beta^{\prime}} \widetilde{E}_{\alpha^{\prime}}+h_{\alpha^{\prime} \beta^{\prime}}(t)\right]
$$


It can be rewritten as

$$
\begin{aligned}
\frac{d\left\langle P_{\alpha \beta}\right\rangle}{d t}= & \frac{1}{i \hbar}\left(E_{\beta}-E_{\alpha}\right)\left\langle P_{\alpha \beta}\right\rangle+\frac{1}{i \hbar} \sum_{\nu}\left(\left\langle P_{\alpha \nu}\right\rangle h_{\beta \nu}(t)-h_{\nu \alpha}(t)\left\langle P_{\nu \beta}\right\rangle\right) \\
& +\sum_{\alpha^{\prime} \beta^{\prime}} K_{\alpha \beta, \alpha^{\prime} \beta^{\prime}}^{h}\left\langle P_{\alpha^{\prime} \beta^{\prime}}\right\rangle,
\end{aligned}
$$

where the generalized relaxation matrix is given by

$$
\begin{aligned}
K_{\alpha \beta, \alpha^{\prime} \beta^{\prime}}^{h}= & \frac{\beta}{i \hbar} \int_{0}^{1} d \lambda\left\langle\left[P_{\alpha \beta}, V\right] e^{-\lambda A} V e^{\lambda A}\right\rangle_{q}+\frac{1}{(i \hbar)^{2}} \int_{0}^{1} d \lambda \\
& \times \int_{-\infty}^{0} d t_{1} e^{\varepsilon t_{1}}\left\langle\left[P_{\alpha^{\prime} \beta^{\prime}}, V\right] e^{-\lambda A}\left[P_{\alpha^{\prime} \beta^{\prime}}\left(t_{1}, t\right), V\left(t_{1}\right)\right] e^{\lambda A}\right\rangle_{q} X_{\alpha^{\prime} \beta^{\prime}}\left(t+t_{1}\right) .
\end{aligned}
$$

Equation (132) gives the generalization of the rate Eq (35) of the Redfieldtype for the case of the external alternating field. A more detailed investigation of this equation and the problem of the evolution of a system in an external field will be carried out separately.

\section{APPENDIX B. CORRELATION FUNCTIONS AND GAUSSIAN APPROXIMATION}

The eigenvalues of the Hamiltonian (80) correspond to well-defined values of $\sum_{i} I_{i}^{z}=I^{z}=m$. Their energy is the sum of a Zeeman energy $m \hbar \omega_{n}$ and a spin-spin energy. A stochastic-theoretical treatment of the spin relaxation phenomena is a useful complementary approach to the consideration of spin evolution. ${ }^{118,119}$ By a stochastic theory it is termed ordinary that kind of theoretical treatment of the problem in which one assumes the random nature of the forces acting on a system. The phenomenon of spin relaxation can be properly interpreted as some stochastic process of spin motion. This stochastic process is determined by the equation of motion of the spin variable. It was formulated ${ }^{33,118}$ plausibly that a Gaussian random process may be well applied for the evolution of the magnetization in the presence of a static external field

$$
\frac{d}{d t} \vec{\mu}=\gamma \vec{\mu} \times\left(\overrightarrow{h_{0}}+\vec{h}\right)
$$

where $\gamma$ denotes the gyromagnetic ratio, $\overrightarrow{h_{0}}$ a static external field, and $\vec{h}$ the fluctuating internal field due to the magnetic moments in the surrounding medium. The effect of the fluctuating internal field $\vec{h}$ is to cause nuclear spin transitions governed by the selection rule $\Delta m= \pm 1$. If the 
Zeeman splitting is small, i.e., $\hbar \omega_{n} \ll k T$, then the transition probability for a $\Delta m= \pm 1$ transition will be proportional to the Fourier transform of correlation functions of the form $\left(h^{+}(t) h^{-}\left(t^{\prime}\right)\right),\left(h^{-}(t) h^{+}\left(t^{\prime}\right)\right),\left(h^{z}(t) h^{z}\left(t^{\prime}\right)\right)$. If we assume the process of $\vec{h}(t)$ to be a Gaussian random process, the problem becomes more easy tractable. From this viewpoint it is reasonable to assume that the equation of spin motion involves the local fluctuating magnetic field whose process is assumed to be a Gaussian random process. ${ }^{118}$

The Gaussian or normal probability distribution law is the limit of the binomial distribution

$$
P(m)=C_{n}^{m} p^{m}(1-p)^{n}
$$

in the limit of large $n$ and $p n(n \rightarrow \infty)$. Here $n$ is the repetition of an experiment, $p$ is the probability of success, and $C_{n}^{m}=n ! / m !(n-m) !$. The normal probability distribution has the form

$$
P(m)=\frac{1}{\sqrt{2 \pi} \sigma} \exp \left(-\frac{1}{2} \frac{\xi^{2}}{\sigma^{2}}\right),
$$

where $\sigma=\sqrt{n p(1-p)}$ is a measure of the width of the distribution. It is clear that the Gaussian distribution results when an experiment with a finite probability of success is repeated a very large number of times. The Gaussian random process is a random process (with discrete or continuous time) which has the normal (Gaussian) probability distribution law for any group of values of the process. The Gaussian random process is determined completely by its average value and correlation function. Thus, the description of the class of Gaussian processes is reduced to the determination of the possible form of the corresponding correlation functions.

Consider now an isotropic distribution of nuclei and rewrite the production of the current operator in Eq. (78) in explicit form

$$
\begin{array}{r}
J(\vec{r}) J\left(\overrightarrow{r_{1}}, t_{1}\right)=\frac{\omega_{n}^{2}}{4} \sum_{k \neq l} \sum_{m \neq n} A_{k l} A_{m n} r_{k l} r_{m n}, \\
\int d \vec{r} \delta\left(\vec{r}-\overrightarrow{r_{k}}\right) \delta\left(\overrightarrow{r_{1}}-\overrightarrow{r_{m}}\right)\left[\operatorname{Tr}\left(I^{z}\right)^{2}\right]^{-1} \operatorname{Tr} I_{k}^{+} I_{l}^{-} I_{m}^{+}\left(t_{1}\right) I_{n}^{-}\left(t_{1}\right) .
\end{array}
$$

To proceed further, the form of the correlation function of nuclear spins in the above expression must be determined. In the theory of NMR the reasonable assumption is that this correlation function can be represented 
in an intuitively understandable way as ${ }^{23,118}$

$$
\begin{aligned}
& \operatorname{Tr}_{k}^{+} I_{l}^{-} I_{m}^{+}(t) I_{n}^{-}(t) \propto \operatorname{Tr}\left(I^{+} I^{-}\right)^{2} f(t) \delta_{k n} \delta_{l m} \exp \left[\frac{i t}{\hbar}\left(\Omega_{l}-\Omega_{k}\right)\right] \\
& \quad=\frac{1}{4} f(t) \delta_{k n} \delta_{l m} \exp \left[\frac{i t}{\hbar}\left(\Omega_{l}-\Omega_{k}\right)\right] .
\end{aligned}
$$

Then the diffusion coefficient $D(\vec{r})$ (78) takes the form

$$
\begin{aligned}
D(\vec{r}) & =\frac{1}{8 \hbar^{2} N(r)} \sum_{k \neq l} A_{k l}^{2} r_{k l}^{2} \delta\left(\vec{r}-\vec{r}_{k}\right) \int_{-\infty}^{\infty} e^{\varepsilon t} d t f(t) \exp \left[\frac{i t}{\hbar}\left(\Omega_{k}-\Omega_{l}\right)\right] \\
& =\frac{1}{8 \hbar^{2}} \sum_{l} A_{r l}^{2} r_{r l}^{2} \int_{-\infty}^{\infty} d t f(t) \exp \left[\frac{i t}{\hbar}\left(\Omega_{r}-\Omega_{l}\right)\right] .
\end{aligned}
$$

The method of moments gives that $f(t)$ is close to the normal probability distribution

$$
f(t)=A \exp \left(-\frac{t^{2} \omega_{d}^{2}}{2}\right), \quad \hbar^{2} \omega_{d}^{2}=\frac{\operatorname{Tr} H_{d i p}^{2}}{\operatorname{Tr}\left(I^{z}\right)^{2}} .
$$

The constant $A$ can be determined from the condition

$$
\int_{-\infty}^{\infty} d t f(t)=1=A \sqrt{\frac{2 \pi}{\omega_{d}^{2}}}, \quad A=\sqrt{\frac{\omega_{d}^{2}}{2 \pi}} .
$$

Thus, we obtain

$$
f(t)=\frac{\omega_{d}}{\sqrt{2 \pi}} \exp \left(-\frac{t^{2} \omega_{d}^{2}}{2}\right) .
$$

For the diffusion coefficient (138) we find

$$
D(\vec{r}) \approx \frac{\omega_{d}}{\hbar^{2} \sqrt{\pi}} \sum_{l} A_{r l}^{2} r_{r l}^{2} \exp \left[-\left(\Omega_{r}-\Omega_{l}\right)^{2} / 4\left(\omega_{d}\right)^{2}\right] .
$$

In the case when $r$ is close to $l$ the frequency difference $\left(\Omega_{r}-\Omega_{l}\right) \gg \omega_{d}$ and $D(\vec{r}) \rightarrow 0$. In the opposite case, when $\left(\Omega_{r}-\Omega_{l}\right) \ll \omega_{d}$ the diffusion coefficient is nearly constant $D(\vec{r}) \sim D$. Thus, we traced back to the notion of the diffusion barrier $\delta$ (95). Consider two neighboring nuclei along the radius from the impurity. The distance between them is equal to the lattice constant $a$. For this case the frequency shift is equal to

$$
\left(\Omega_{\delta}-\Omega_{\delta+a}\right) \approx \omega_{d},
$$


where $\omega_{d} \approx 6 \gamma_{n}^{2} \hbar a^{-3}$. Consider this constraint more carefully. We have

$$
\begin{aligned}
\gamma_{n} \gamma_{M} \hbar\left\langle S^{z}\right\rangle\left\{\frac{1+B \cos \left(\delta k_{F}+\phi_{B}\right)}{\delta^{3}}-\frac{1+B \cos \left((\delta+a) k_{F}+\phi_{B}\right)}{(\delta+a)^{3}}\right\} \\
=\gamma_{n} \gamma_{M} \hbar\left\langle S^{z}\right\rangle\left\{\frac{1+B \cos \left(\delta k_{F}+\phi_{B}\right)}{\delta^{3}}-\frac{1-B \sin \left(\delta k_{F}+\phi_{B}\right) a k_{F}}{\delta^{3}}\right. \\
\left.\quad+\frac{B \cos \left(\delta k_{F}+\phi_{B}\right)}{\delta^{3}}+3 \frac{1+B \cos \left(\delta k_{F}+\phi_{B}\right)}{\delta^{4}} a\right\} \\
=\frac{a}{\delta^{3}} \gamma_{n} \gamma_{M} \hbar\left\langle S^{z}\right\rangle\left\{3 \frac{1+B \cos \left(2 \delta k_{F}+\phi_{B}\right)}{\delta}-B \sin \left(\delta k_{F}+\phi_{B}\right) k_{F}\right\}=6 \gamma_{n}^{2} \hbar a^{-3}
\end{aligned}
$$

For the rough estimation we omit the cos and sin contributions. Then we obtain

$$
6 \gamma_{n}^{2} \hbar a^{-3}=\gamma_{n} \gamma_{M} \hbar\left\langle S^{z}\right\rangle \frac{a}{\delta^{4}}, \quad \delta=a \sqrt[4]{\left[\frac{\gamma_{M}}{\gamma_{n}}\left\langle S^{z}\right\rangle\right]} .
$$

\section{REFERENCES}

1. H. B. G. Casimir and F. K. Du Pre, Physica 5, 507 (1937).

2. H. B. G. Casimir, Physica 6, 156 (1939).

3. F. Bloch, Phys. Rev. 70, 460 (1946).

4. C. J. Gorter, Paramagnetic Relaxation, Elsevier, Amsterdam, New York (1947).

5. N. Bloembergen, Physica 15, 386 (1949).

6. R. Kubo and K. Tomita, J. Phys. Soc. Jpn 9, 888 (1954).

7. A. G. Redfield, Phys. Rev. 101, 67 (1956).

8. A. G. Redfield, IBM J. Res. Develop. 1, 19 (1957).

9. A. G. Redfield, Phys. Rev. 116, 315 (1959).

10. L. C. Hebel and C. P. Slichter, Phys. Rev. 113, 1504 (1959).

11. W. J. Caspers, Physica 26, 778, 798 (1960).

12. A. Sher and H. Primakoff, Phys. Rev. 119, 178 (1960).

13. A. Sher and H. Primakoff, Phys. Rev. 130, 1267 (1963).

14. N. Bloembergen, Nuclear Magnetic Relaxation, W. A. Benjamin, New York (1961).

15. J. R. Klauder and P. W. Anderson, Phys. Rev. 125, 912 (1962).

16. J. Korringa, Phys. Rev. 133, A1228 (1964).

17. J. Korringa, J. L. Motchane, P. Papon, and A. Yoshimori, Phys. Rev. 133, A1230 (1964).

18. S. Stenholm and D. ter Haar, Physica 32, 1361 (1966).

19. W. J. Caspers, Theory of Spin Relaxation, Interscience Publication, New York (1964).

20. R. L. Strombothe and E. L. Hanh, Phys. Rev. 133, A1616 (1964).

21. P. N. Argyres, in Proc. Eindhoven Conf. on Magnetic and Electric Resonance and Relaxation, J. Smidt, eds., North-Holland, Amsterdam, (1963), p. 555.

22. P. N. Argyres and P. L. Kelley, Phys. Rev. 134, A98 (1964).

23. L. L. Buishvili and D. N. Zubarev, Solid State Phys, 7, 722 (1965).

24. G. Sauermann, Physica 32, 2017 (1966).

25. A. G. Redfield, In: Advance Magnetic Resonance, J. S. Waugh eds., Academic Press, New York (1966), Vol. 1, p.1.

26. L. J. Lowe and S. Gade, Phys. Rev. 156, 817 (1967). 
27. B. Robertson, Phys. Rev. 153, 391 (1967).

28. J. Jeener, in Advance Magnetic Resonance, J. S. Waugh eds., Academic Press, New York (1968), Vol. 3, p.206.

29. P. R. Moran, J. Phys. Chem. Solids, 30, 297 (1969).

30. H. Meyer, S. M. Myers, and J. P. Remeika, J. Phys. Chem. Solids, 30, 2687 (1969).

31. H. Meyer, S. M. Myers, and J. P. Remeika, J. Phys. Chem. Solids, 32, 867 (1971).

32. M. Goldman, Spin Temperature and Nuclear Magnetic Resonance in Solids, Oxford University Press, Oxford, New York (1970).

33. A. Abragam, Principles of Nuclear Magnetism, Oxford University Press, Oxford, (1961).

34. D. Wolf, Spin-temperature and Nuclear-spin Relaxation in Matter. Oxford University Press, Oxford, New York (1979).

35. C. P. Slichter, Principles of Magnetic Resonance, Springer, Berlin, (1980).

36. G. J. Ehnholm, J. P. Ekström, J. F. Jacquinot, M. J. Loponen, O. V. Lounasmaa, and J. K. Soini, J. Low Temp. Phys. 39, 417 (1980).

37. A. Abragam and M. Goldman, Nuclear Magnetism: Order and Disorder, Oxford University Press, Oxford, New York (1982).

38. G. S. Boutis, D. Greenbaum, H. Cho, D. G. Gory, and C. Ramanathan, Phys. Rev. Lett. 92, 137201 (2004).

39. W. K. Rhim, A. Pines, and J. S. Waugh, Phys. Rev. Lett. 25, 218 (1970).

40. L. L. Buishvili, Zh. Exp. Th. Phys., 49, 1868 (1965).

41. L. L. Buishvili, Solid State Phys, 7, 1871 (1965).

42. N. S. Bendiashvili, L. L. Buishvili, and M. D. Zviadadze, Solid State Phys. 8, 2919 (1966).

43. L. L. Buishvili and M. D. Zviadadze, Phys.Lett. A 25, 86 (1967).

44. L. L. Buishvili and M. D. Zviadadze, Solid State Phys. 10, 2553 (1968).

45. P. Borckmans and D. Walgraef, in Proc. XV Colloque Ampere, North-Holland, Amsterdam (1969), p. 418.

46. L. L. Buishvili and N. P. Giorgadze, Dokl Akad Nauk SSSR 189, 508 (1969).

47. L. L. Buishvili, M. D. Zviadadze, and G. R. Khutsishvili, Zh. Exp. Th. Phys, 56, 290 (1969).

48. L. L. Buishvili and N. P. Giorgadze, Solid State Phys 12, 1817 (1970).

49. N. S. Bendiashvili, L. L. Buishvili, and M. D. Zviadadze, Zh. Exp. Th. Phys, 58, 597 (1970).

50. L. L. Buishvili and N. P. Giorgadze, Dokl Acad Nauk SSSR 191, 58 (1970).

51. N. G. Fazleev, Sov. J. Low Temp. Phys. 5, 181 (1979).

52. N. G. Fazleev, Sov. J. Low Temp. Phys. 6, 693 (1980).

53. D. Tayurskii, Phys. Stat. Sol. B 162, 545 (1989).

54. R. Nigmatullin and D. Tayurskii, Physica A 175, 275 (1991).

55. V. Romero-Rochin, A. Orsky, and I. Oppenheim, Physica A 156, 244 (1989).

56. W. T. Grandy, Foundations of Statistical Mechanics: Equilibrium Theory, D. Reidel Publishing Doddrecht, Holland (1987), Vol. 1.

57. W. T. Grandy, Foundations of Statistical Mechanics: Nonequilibrium Phenomena, D. Reidel Publishing (Doddrecht Holland, (1988), Vol. 2

58. M. Toda, R. Kubo and N. Saito, Statistical Physics: Equilibrium Statistical Mechanics, Springer Publishing Berlin, (1992), Vol. 1.

59. R. Kubo, M. Toda, and N. Hashitsume, Statistical Physics: Nonequilibrium Statistical Mechanics, Springer Publishig Berlin, (1991), Vol. 2.

60. D. N. Zubarev, Nonequilibrium Statistical Thermodynamics, Consultant Bureau, New York (1974).

61. J. A. McLennan Introduction to Nonequilibrium Statistical Mechanics, Prentice Hall, New Jersey, (1989).

62. R. Zwanzig, Nonequilibrium Statistical Mechanics, Oxford University Press, Oxford, New York (2001).

63. J. L. Lebowitz, Physica A 263, 516 (1999).

64. A. L. Kuzemsky, Intern. J. Modern Phys. B 19, 1029 (2005) (cond-mat/0502194). 
65. N. N. Bogoliubov, in Studies in Statistical Mechanics, J. de Boer and G. E. Uhlenbeck, eds., North-Holland, Amsterdam, (1962), Vol. 1, p.1.

66. N. N. Bogoliubov, On the stochastic processes in the dynamical systems", Communications E17-10514, JINR, Dubna (1977).

67. Z. Onyszkiewicz, Physica A 143, 287 (1987).

68. M. Gell-Mann and M. L. Goldberger, Phys. Rev. 91, 398 (1953).

69. N. N. Bogoliubov, Quasi-averages in the problems of statistical mechanics, Communications D-781, JINR, Dubna (1961).

70. L. van Hove, Physica 23, 411 (1957).

71. R. Zwanzig, Physica 30, 1109 (1964).

72. A. Fulinski and W. J. Kramarczyk, Physica 39, 575 (1968).

73. L. A. Pokrowsky, Dokl Acad Nauk SSSR 183, 806 (1968).

74. R. I. Cukier and J. M. Deutch, J. Chem. Phys. 50, 36 (1969).

75. P. Hubbard, Rev. Mod. Phys. 33, 249 (1961).

76. G. R. Khutsishvili, Usp. Fiz. Nauk. 87, 211 (1965); Sov. Phys. Uspekhi, 8, 743 (1966).

77. G. R. Khutsishvili Comment Solid State Phys. 5, 23 (1972).

78. P. M. Morse and H. Feshbach, Methods of Theoretical Physics, McGraw-Hill Book Co., New York (1953).

79. G. Arfken, Mathematical Methods for Physicists, Academic Press New York (1985).

80. J. P. Stark, Solid State Diffusion, Wiley, New York (1976).

81. W. E. Blumberg, Phys. Rev. 119, 79 (1960).

82. D. A. Rigney and C. P. Flynn, Phil. Mag. 15, 1213 (1967).

83. E. C. Hirschkoff, O. G. Symko, and J. C. Wheatley, J. Low Temp. Phys. 5, 155 (1971).

84. J. J. Peters and C. P. Flynn, Phys. Rev. B 6, 3343 (1972).

85. K. Yoshida and T. Moriya, J. Phys. Soc. Jpn 11, 33 (1956).

86. W. Kohn and S. H. Vosko, Phys. Rev. 119, 912 (1960).

87. M. B. Walker, Phys. Rev. 176, 432 (1968).

88. B. Giovannini and A. J. Heeger, Helv. Phys. Acta 42, 639 (1969).

89. J. Solyom, Z. Phys. 238, 195 (1970).

90. M. B. Walker, Phys. Rev. B 1, 3690 (1970).

91. B. Giovannini, P. Pincus, G. Gladstone, and A. J. Heeger, J. Physique 32, C1-163 (1971).

92. J. Zitkova-Wilcox, Solid State Commun. 12, 1109 (1973).

93. P. Nozieres, J. Low Temp. Phys. 17, 31 (1974).

94. N. Bloembergen and T. J. Rowland, Acta Metall. 1, 731 (1953).

95. N. Bloembergen and T. J. Rowland, Phys. Rev. 97, 1679 (1955).

96. T. J. Rowland, Phys. Rev. 119, 900 (1960).

97. R. L. Streever, Phys. Rev. 134, A1612 (1964).

98. S. Clough and W. I. Goldburg, J. Chem. Phys. 45, 4080 (1966).

99. J. R. Asik, M. A. Ball, and C. P. Slichter, in Magnetic Resonance and Relaxation, Proc. XIV Colloque Ampere, Ljubljana, 1966 R. Blinc, eds., North-Holland, Amsterdam (1967), p. 448.

100. O. J. Lumpkin, Phys. Rev. 164, 324 (1967).

101. A. C. Gossard, A. J. Heeger, and J. H. Wernick, J. Appl. Phys. 38, 1251 (1967).

102. R. E. Walstedt, R. C. Sherwood, and J. H. Wernick, J. Appl. Phys. 39, 555 (1968).

103. R. E. Walstedt and A. Narath, Phys. Rev. B 6, 4118 (1972).

104. M. I. Aaalto, P. M. Berglund, H. K. Collan, G. J. Ehnholm, R. G. Gylling, and O. V. Lounasmaa, Physica 61, 314 (1972).

105. D. C. Lo, D. V. Lang, J. B. Boyce, and C. P. Slichter, Phys. Rev. B 8, 973 (1973).

106. H. Alloul and P. Bernier, J. Phys. F: Metal Phys. 4, 870 (1974).

107. H. Alloul, J. Darville, and P. Bernier, J. Phys. F: Metal Phys. 4, 2050 (1974).

108. P. Bernier and H. Alloul, J. Phys. F: Metal Phys. 6, 1193 (1976).

109. R. E. Walstedt and L. R. Walker, Phys. Rev. B 9, 4857 (1974).

110. Y. Yafet and V. Jaccarino, Phys. Rev. 133, A1630 (1964).

111. M. A. Ruderman and C. Kittel, Phys. Rev. 96, 99 (1954). 
112. A. L. Kuzemsky, Physica B 355, 318 (2005).

113. J. H. Van Vleck, Phys. Rev. 74, 1168 (1948).

114. B. Coqblin The Electronic Structure of Rare-Earth Metals and Alloys: the Magnetic Heavy Rare-Earths, Academic Press New York, (1977).

115. F. Lado, J. D. Memory and G. W. Parker, Phys. Rev. B 4, 1406 (1971).

116. J. Korringa, Physica 16, 601 (1950).

117. T. Moriya, J. Phys. Soc. Jpn . 18, 516 (1963).

118. R. Kubo, in Fluctuation, Relaxation, and Resonance in Magnetic Systems Proc. Scottish Universities Summer School, 1961, D. ter Haar, ed., Oliver and Boyd, Edinburgh (1962), p. 23.

119. R. Kubo, Hyperfine Interactions 8, 731 (1981). 